SPECTRUM OF IC 5117

\title{
Optical Spectrum of the compact Planetary Nebula IC 5117
}

\author{
Siek Hyung ${ }^{1}$, Lawrence H. Aller ${ }^{2}$, Walter A. Feibelman ${ }^{3}$, Seong-Jae Lee ${ }^{4}$ \\ ${ }^{1}$ Korea Astronomy Observatory, 61-1 Whaam-dong, Yusong-gu, TaeJon 305-348, Korea \\ ${ }^{2}$ Physics and Astronomy Department, University of California, Los Angeles, California 90095, \\ USA \\ ${ }^{3}$ Laboratory for Astronomy and Solar Physics, Code 684.1, NASA Goddard Space Flight Center, \\ Greenbelt, MD 20771, USA \\ ${ }^{4}$ Dept. of Astronomy and Space Science, Chungnam National University, TaeJon 305-764, Korea
}

\begin{abstract}
High resolution spectroscopic data of the very compact planetary nebula IC 5117 are obtained in the optical wavelengths, $3700 \AA-10050 \AA$, with the Hamilton Echelle Spectrograph at Lick Observatory, and which have been analyzed along with the International Ultraviolet Explorer (IUE) UV archive data. Although a diagnostic diagram shows significant density and temperature fluctuations, our analysis indicates that the nebular gas may be represented by a homogeneous shell of extremely high density gas, $N_{\epsilon} \sim 90000 \mathrm{~cm}^{-3}$. The average electron temperatures, e.g. indicated by the [O III] diagnostics, are around $12000 \mathrm{~K}$. We construct a photoionization model to represent most of the observed line intensities, and the physical condition of this compact nebulosity. Based on the semi-empirical ionization correction approach, and model indications, we derived the elemental abundances: $\mathrm{He}, \mathrm{C}, \mathrm{N}, \mathrm{O}, \mathrm{Ne}$, and $\mathrm{Ar}$ appear to be normal or marginally depleted compared to the average planetary nebula, while the remaining elements, $\mathrm{S}, \mathrm{Cl}$, and $\mathrm{K}$ appear to be enhanced. IC 5117 is perhaps a very young compact planetary nebula, slightly more evolved than the other well-known compact planetary nebula IC 4997. The central stellar temperature is likely to be around $120000 \mathrm{~K}$, evolved from a C-rich AGB progenitor.
\end{abstract}

Subject headings: ISM: abundances: planetary nebulae: individual (IC 5117)

\section{Introduction}

IC 5117 is a very young compact planetary nebula (PN) with molecular emission. CO often succeeds in forming and surviving within the envelopes of more massive nebulae (Mamon et al. 1988). Gussie \& Taylor (1995) observed both H I and CO envelopes in IC 5117: they suggested that the $\mathrm{H} \mathrm{I}$ is formed within a photodissociation region inside a larger molecular envelope and 
exterior to the ionized gas. The spatial distributions of the $\mathrm{CO}$ and $\mathrm{H} \mathrm{I}$ are unknown, so a spatial comparison between the molecular and atomic components is impossible.

In the radio continuum and $\mathrm{H} 92 \alpha$ study by Miranda et al. (1995), strong variations of the radial velocity were detected on angular scales of $\simeq 0.3^{\prime \prime}$, and a kinematical age of $\simeq 350$ yr was inferred (for an assumed distance of $3 \mathrm{kpc}$ ). They also reported variations of the electron temperature and density on scales of $\simeq 0.4^{\prime \prime}$. The high brightness temperature in radio frequencies, which is indicative of distance-independent density information, had been noted in earlier studies, e.g. the VLA 6-cm continuum observation by Zhang (1995). IC 5117 is known to be one of the youngest PNe. It is unknown whether or not the observed neutral gas is causally connected with the nebula, and it is not clear whether the ionized shell in IC 5117 grows at the expense of an outer neutral shell.

With the Image Tube Scanner (ITS), Aller and Czyzak (1979, hereafter AC79) secured a number spectral lines, but the wavelength dispersion was relatively poor compared with the currently available high dispersion spectral data obtained with charge coupled devices (CCDs) used with, e.g. an echelle spectrograph. Thus, it would be better to find the abundances from recently available high quality data, with the help of appropriate models. We revisited IC 5117 to secure a high dispersion optical spectrum, from 3700 to $10050 \AA$, with the Hamilton Echelle Spectrograph (HES) at Lick Observatory. We compared the optical spectrum from the HES with that of the ITS, and we also re-measured the IUE archive data in the UV region. For these relatively complete wavelength coverage data, we obtain the diagnostics from which we compute the ionic concentrations. With the help of a reliable photoionization model, we try to fit the observed optical emission lines and other wavelength region archival data to determine the physical condition of IC 5117 which best represents the diagnostics and the nebular gas. Finally, we determine the abundances of IC 5117, compare these with the solar and average nebular abundance, and discuss briefly the evolutionary status. Table 1 gives some basic data for IC 5117, and useful references.

\section{Observations}

There are 4 IUE spectra available for IC 5117: SWP 25835 (30min, 1985 May 2), SWP 31825 (150min, 1987 Sep 11), LWP 05883 (30min, 1985 May 2), and LWP 05884 (295min, 1985 May 2). All these spectra are low dispersion, and were taken through the large $\left(10^{\prime \prime} \times 23^{\prime \prime}\right.$ oval $)$ entrance aperture of the IUE cameras. The IUE data were reduced with the latest IUE reduction techniques at the Goddard Space Flight Center (GSFC), i.e. with the NEWSIPS routine. The apparent size of IC 5117 is small enough to fit into the large entrance aperture, so that the IUE-observed emission comes from the entire nebula. We measured the spectrum from two long exposures, i.e. SWP 31825 and LWP 05884 , and ignored the other relatively poor quality exposures. These emission line measurements are given in Table 2. Successive columns of Table 2 give the observed and laboratory wavelengths, the ion, Seaton's extinction parameter, $k_{\lambda}$, the extinction corrected intensity with $\mathrm{E}(\mathrm{B}-\mathrm{V})=0.88$ [relative to $\mathrm{I}(\mathrm{H} \beta)=100$ ], and the measured flux in units of $10^{-14}$ erg $\mathrm{cm}^{-2} \mathrm{~s}^{-1}$ (note that the flux unit is one order of magnitude lower than those of the other 
well-known PNe investigated in our previous studies!). In Fig. 1 we plot the combined IUE SWP + LWP spectra in the wavelength range from 1200 to $3250 \AA$ (the extinction correction was not applied). All spectra were smoothed with a 3 -point running average.

For the near UV region (from the limit of the Balmer series down to the atmospheric cutoff near $3100 \AA$ ), we refer to the high sensitivity 'green' tube (ITS) archive data by Likkel and Aller (1986, hereafter LA86). Table 3 gives the near UV measurements by Likkel (private communication). The first column gives the measured wavelength from LA86; the second column gives the laboratory identification; the extinction parameter, $k_{\lambda}$, is listed in column (3). Column (4) gives the derived intensity, corrected for interstellar extinction; column (5) gives the measured flux data secured by LA86 from the green tube ITS observations. Here, the intensities are given on the scale of $\mathrm{I}(\mathrm{H} \beta)$ $=100$, after applying the extinction correction (with $C=1.29$ ), while the fluxes are in units of $10^{-14} \mathrm{erg} \mathrm{cm}^{-2} \mathrm{~s}^{-1}$. ' $\mathrm{B}$ ' in the last column denotes Bowen fluorescent $\mathrm{O}$ III lines, which had been investigated by LA86. In this region fall lines of $\mathrm{He} I$ and $\mathrm{O}$ III, but no lines of He II, O IV, [Ne III] (auroral-type), [Ne V], and [Na IV]. We quoted the [OII]3727 lines in order to compare with the HES spectral measurements. In Table 3, and in the following Tables, we have given 1 or 2 more significant figures than the data justify, to avoid round-off errors.

The optical region observations were all obtained with the HES at the Coudé focus of the $3 \mathrm{~m}$ Shane telescope of Lick Observatory, on 1995 August 18. We obtained two exposures, 120 minutes and 5 minutes, on IC 5117. The sky was very clear during our observations, and seeing was less than $\sim 1.5^{\prime \prime}$. The spectroscopic slit employed was $640 \mu \mathrm{m}$ in width, which amounted to $\sim 1.2^{\prime \prime}$ in image size at the Coude focus. For this slit width, the limiting resolution on the CCD chip was about 2 pixels which amounted to $0.05 \AA$ wavelength dispersion at $3600 \AA$, and increased to $0.15 \AA$ at $8850 \AA$. The slit length of $4^{\prime \prime}$ was chosen to avoid confusion of successive echelle orders.

For spectral calibration, we took exposures of a Th-Ar arc lamp to set the wavelength dispersion scale; a dome-quartz lamp to fix a flat field which allowed us to correct for pixel to pixel sensitivity fluctuations; and finally exposures on two standard stars of known energy distribution, i.e. M3923 (chosen for the blue wavelength region flux calibration) and Eta Ursa Majoris (for the red wavelength region flux calibration). The absolute fluxes of these two standard stars were available from Mr. Remington Stone (private communication, 1995). We used a large $2048 \times 2048$ pixel CCD, which covered the whole HES echelle pattern. The reduction procedures are described in Hyung (1994). We present the HES results in Table 4. A large number of optical lines were measured. Successive columns give: the measured wavelength (corrected for radial velocity), the wavelength of the most probable identification, the ion, the multiplet number from Moore's tabulations (1974, 1993), and Seaton's extinction parameter, $k_{\lambda}$. We found the radial velocity of IC 5117 to be $-38.7 \pm 0.7 \mathrm{~km} \mathrm{~s}^{-1}$, while Acker et al. (1992) quote $-26.1 \pm 1.3 \mathrm{~km} \mathrm{~s}^{-1}$. The discrepancy may be caused by some of the selected lines being different between these two analyses. We obtained our value from a comparison of the observed wavelengths of the strong lines $(I>1.0)$ with the laboratory wavelengths, using the least squares method; the central wavelengths of the emission profile were converted to heliocentric radial velocity $\left(+6.5 \mathrm{~km} \mathrm{~s}^{-1}\right)$, following the method of Herrick (1935). 
The 6th column gives the HES intensity on the scale $\mathrm{I}(4861)=100.0$, corrected for interstellar extinction, with an extinction coefficient $C=\log \mathrm{I}(\mathrm{H} \beta) / \mathrm{F}(\beta)=1.40 \pm 0.13$ found from Balmer line ratios, such as $F(H \alpha) / F(H \beta)$, and from a comparison of Balmer and Paschen lines of the same upper quantum number: $\mathrm{n}=10,12,14, \& 16$, i.e. $9015 \AA$ vs. $3798 \AA$ and $8750 \AA$ vs. $3750 \AA$ etc; this is different from the value of $C=1.29$ used for the UV region IUE and near-UV region ITS data. The higher value of $C$ for the HES data is probably an overestimation due to an observational or data reduction error involving the instrumental and atmospheric response functions. The 7 th column presents the HES flux on the scale $F(4861)=100.0$, while the last column lists the formal root mean square (RMS) \% error, as deduced from the internal disagreement of measurements made with different CCD chip position settings (whenever two or more measurements are available).

The spectrum of IC 5117 was not divided by that of the standard star in order to take out the 1st order effects of the atmosphere. However, since the HES produces a high dispersion spectrum, one can clearly tell which lines are severely affected by the telluric absorption. The errors increase towards the ends of each order, and with the underlying noise. Lines affected by "bleeding" from a strong line in a nearby order may be seriously impacted. By taking a graded series of exposures, this difficulty can often be overcome. Several procedures are available for estimating the accuracy of the measurements. By comparing data obtained on different nights, and with different chip positions, we can assess the effect of guiding errors, influence (if any) of position in the field, and of the response function. On the absolute flux scale Flux $(\mathrm{H} \beta)=100$, statistics show that lines weaker than $\mathrm{I}=1.0$ will have errors of $15 \%$ to $40 \%$; for lines in the range $1.0 \leq \mathrm{I} \leq 10.0$, errors fall in the interval $7 \%$ to $20 \%$; for stronger lines, we estimate errors of $3 \%$ to $10 \%$. In Fig. 2, we present three reduced spectral scans to show the quality of our HES data.

\section{Diagnostics}

Numerous lines, including many strategically important diagnostic lines especially useful for nebular diagnostic and abundance determination, are observed in the optical spectrum of IC 5117. All of the listed optical lines in Table 4 were resolved, but their line profiles mostly do not show a double peak feature. However, we were able to separate the double component for the case of strongly measured low excitation lines, i.e. [N II]6584, using the STARLINK/DIPSO tool. The derived expansion velocity from the $\left[\mathrm{N}\right.$ II] line profiles is about $11.4 \mathrm{~km} \mathrm{~s}^{-1}$, while expansion velocities quoted by Acker et al. (1992), i.e. $21.5 \mathrm{~km} \mathrm{~s}^{-1}$ ([N II]) and $16.5 \mathrm{~km} \mathrm{~s}^{-1}$ ([O III]), are larger than our derivation. The following ions are detected in the HES spectrum of IC 5117: $\mathrm{H}$, He I, He II, C I, [C I], C II, C III, C IV, N I, [N I], N II, [N II], N III, [O I], O II, [O II], O III, [O III], Ne I, [Ne III], [Ne IV], S I, S II, [S II], [S III], [Cl II], [Cl III], [Cl IV], [Ar III], [Ar IV], [Ar V], [K III], [K IV], [Ca VII]?, Mn I?, [Mn V], [P I], [F II]?, [Fe II], [Fe III], [Fe VI], and [Fe VII]. UV and near-UV lines detected are as follows: He I, He II, C II], C III], C IV, [N II], N III], N IV, N V?, [O II], O III, O III], [Ne IV], [Ne V], Si II], Si III], Ar II, Na IV. Diagnostic line ratios suitable for fixing the electron densities and temperatures, $\left(N_{\epsilon}, T_{\epsilon}\right)$, are listed in Table 5 , and Fig. 3 shows 
the diagnostics based on those line ratios involving equivalent $\mathrm{p}^{2}$ and $\mathrm{p}^{4}$ electrons. Electronic collision strengths involving the plasma and nebular diagnostics are constantly updated from the most recently available data, as in our previous investigations, e.g. Hyung et al. (2001).

Diagnostics of [Cl III], [S II], [N II], [Ar III], and [Cl IV] intersect near a single point, i.e. $\log N_{\epsilon}$ $=4.6$ and $T_{\epsilon} \sim 12000 \mathrm{~K}$. However, the electron temperatures, especially indicated by the [O III] lines, are extremely high, for the above density, $\log N_{\epsilon}=4.6$. According to Aller \& Liller (1968: see their Fig. 1), the $\mathrm{He} I 14686 / \mathrm{H} \beta$ ratio $\sim 0.1$ implies that this $\mathrm{PN}$ has excitation class 6 . The other line ratio $\mathrm{I}(\lambda 3726+3729) / \mathrm{I}(\lambda 4959)$ also indicates excitation class 6 . Providing this $\mathrm{PN}$ is in a medium excitation class, we may be able to choose another point at a much higher density $\log N_{\epsilon}=4.95$ (corresponding to $N_{\epsilon}=90000 \mathrm{~cm}^{-3}$ ) as a representative physical condition. This latter point implies relatively lower electron temperatures, e.g. $\mathrm{T}_{\epsilon} \simeq 11800 \mathrm{~K}$ from the [O III] $[4959+5007] / 4363 \mathrm{ratio}$, and $\mathrm{T}_{\epsilon} \sim 11500 \mathrm{~K}$ from [Ar III] and [Cl $\left.\mathrm{IV}\right]$. In this case, the electron temperatures for the lower excitation line-emitting strata, e.g. [S II], [N II], would also be lower, e.g. $\mathrm{T}_{\epsilon}([\mathrm{N} \mathrm{II}]) \sim 9000 \mathrm{~K}$. For $\left[\mathrm{S} \mathrm{III]}\right.$ and $[\mathrm{O} \mathrm{II}]$, we still find very high electron temperatures, $\mathrm{T}_{\epsilon}$ $\sim 14500 \mathrm{~K}$. Model investigations presented in the following section also seem to indicate physical conditions in favor of the relatively high density nebula gas. In fact, the nebula itself may consist of many inhomogeneous blobs and filaments, where some effects of $\mathrm{T}_{\epsilon}$ fluctuation, considerably greater than that predicted by our photoionization models, may exist (see Peimbert et al. 1995).

With the forbidden lines involving $\mathrm{p}^{3}$ electrons, one can also obtain diagnostics for both density and temperature at the same time (see Keenan et al. 2000; 1999; 1997; 1996). (1) [O II]: $\lambda 3729 / \lambda 3726$ vs. $\lambda 7320 /(\lambda 3726+\lambda 3729)$ gives $\left(\mathrm{T}_{\epsilon}, \log N_{\epsilon}\right)=(12500 \mathrm{~K}, 4.9)$, while $\lambda 3729 / \lambda 3726$ vs. $\lambda 7330 /(\lambda 3726+\lambda 3729)$ gives $(15000 \mathrm{~K}, 4.8)$. Similarly, (2) [Ar IV]: $\lambda 4711 / \lambda 4740$ vs. $\lambda 7263 /(\lambda 4711+\lambda 4740)$ gives $\left(\mathrm{T}_{\epsilon}, \log N_{\epsilon}\right)=(18000 \mathrm{~K}, 4.9) ;(3)[\mathrm{S} \mathrm{II}]: \lambda 6716 / \lambda 6731$ vs. $\lambda 4068 /(\lambda 6716+\lambda 6731)$ gives $\left(\mathrm{T}_{\epsilon}, \log N_{\epsilon}\right)=(15000 \mathrm{~K}, 4.4)$, while $\lambda 6716 / \lambda 6731$ vs. $\lambda 4076 /(\lambda 6716$ $+\lambda 6731)$ gives $(17500 \mathrm{~K}, 4.4)$. (4) [Cl III]: $\lambda 5518 / \lambda 5538$ vs. $\lambda 8434 /(\lambda 5518+\lambda 5538)$ gives $\left(\mathrm{T}_{\epsilon}\right.$, $\left.\log N_{\epsilon}\right)=(20000 \mathrm{~K}, 4.6)$. The electron temperatures indicated by these $\mathrm{p}^{3}$ diagnostics are probably subject to a relatively large error (due to the crowding of temperature diagnostic lines, see e.g. Figs. $2 \& 3$ of Keenan et al. 1996), so the temperature determination from the $\mathrm{p}^{3}$ diagnostics may incur a large error. On the other hand, the density diagnostics appear to be quite useful. For most ions, the electron densities indicated by the $\mathrm{p}^{3}$ diagnostic lines are $N_{\epsilon}=90000 \mathrm{~cm}^{-3}\left(\log N_{\epsilon}\right.$ $=4.9$ ), but for some other ions, e.g. [S II] and [Cl III], lower values are indicated, i.e. $N_{\epsilon} \sim 30000$ $-40000 \mathrm{~cm}^{-3}\left(\log N_{\epsilon}=4.4-4.6\right)$. The neutral lines, such as [N I], must be formed in a region of very low density, $N_{\epsilon} \sim 6300 \mathrm{~cm}^{-3}$.

\section{Theoretical Models}

To construct a theoretical model, one must know the spectral energy distribution (SED) of the central star of the planetary nebula (CSPN), or else certain other properties of the CSPN. The SED of the CSPN can be calculated by employing Hubeny's model atmosphere (1988). The CSPN of IC 
5117 is classified as a Wolf-Rayet (WR) star. Köppen \& Tarafdar (1978) derived the temperature of the CSPN, i.e. $64000 \mathrm{~K}$ from the $\left[\mathrm{O}^{2+} / \mathrm{O}^{+}\right.$ratio] and $67000 \mathrm{~K}$ from the $[\mathrm{He}(4686) / \mathrm{H} \beta$ ratio], while Zhang \& Kwok (1992) derived a similar CSPN temperature of $56700 \mathrm{~K}$, from their model fitting to the IR lines, and to the observed continuum SED. We directly applied Hubeny's theoretical model atmospheres based on some of the selected properties of the CSPN (i.e. $\mathrm{T}_{e f f}$, stellar radius, $\log \mathrm{g}$ ), to the photo-ionization modeling, until it gave a correct level of nebular excitation (using the energy-balance method and the Zanstra method), and the correct electron temperatures. From our trials, we found that model atmospheres with relatively high temperatures are suitable for the CSPN, e.g. $\mathrm{T}_{e f f}=120000 \mathrm{~K}$. The model predictions with lower temperatures for the CSPN, e.g. $\mathrm{T}_{\text {eff }} \sim 85000 \mathrm{~K}$, seem to fit some of lines, but the predicted electron temperatures are in general too low.

Details of parameters adopted in our model are given in Table 6 (see Model B in Table 7). Acker et al. (1992) quoted various distance determinations to IC 5117, which together show a large scatter, from 0.8 to $7.78 \mathrm{kpc}$. To a first approximation, the correct value of the distance to the $\mathrm{PN}$ is not critical in fitting the line intensities. However, we must narrow it down to a reasonable range, since only the correct distance would give an appropriate physical scale for both the CSPN and the PN. We adopted an intermediate value of $\sim 3.0 \mathrm{kpc}$. Thus, for a distance of $3.0 \mathrm{kpc}$, we refined the model, scaling the CSPN properties and model geometry to reproduce the absolute $\mathrm{H} \beta$ flux to within observational errors.

The absolute intrinsic $\mathrm{H} \beta$ flux is $\mathrm{F}_{\text {corr }}(\mathrm{H} \beta)=8.33-10.7 \times 10^{-11}$ [written as $(-11)$, henceforth] erg $\mathrm{cm}^{-2} \mathrm{~s}^{-1}$ for $C=1.29-1.4$ (from the observed $\mathrm{H} \beta$ flux, $\mathrm{F}(\mathrm{H} \beta)=4.27(-12) \mathrm{erg} \mathrm{cm}^{-2} \mathrm{~s}^{-1}$, see Tables 1 and 6$)$. The model predicts $F(H \beta)=9.67(-11) \mathrm{erg} \mathrm{cm}^{-2} \mathrm{~s}^{-1}$. The observed visual magnitude is $m_{v}=16.7$, and accordingly the intrinsic visual magnitude $\mathrm{V}_{o b s}=14.0$ using $\mathrm{E}_{B-V}=$ 0.88 (the corresponding total extinction $A_{v}$ is here taken as $3.1 \mathrm{E}_{B-V}$ ). The model predictions give values that are about one magnitude lower than the observed ones: $V_{\text {pred }}=15.1$ and $B_{\text {pred }}=14.9$. In addition, the model cannot match the observed nebular size: the outer radius of the model shell is density bounded, and its projected angular scale on the sky is slightly larger than the observed one.

The CSPN energy distribution used in the model has $\mathrm{T}_{\text {eff }}=120000 \mathrm{~K}$ and $\log \mathrm{g}=8.5$, with $\mathrm{He} / \mathrm{H}=0.1$, and with a nebular heavy element distribution in the central star. The nebula is assumed to be a homogeneous shell with $N_{\mathrm{H}}=80000 \mathrm{~cm}^{-3}$. No filling factor is introduced in the shell gas. We assume a central star radius of $R_{*}=0.06 R_{\odot}$ and, as a result, $L_{*}=670 \mathrm{~L}_{\odot}$. A significant amount of the energy is emitted in the far-infrared due to the thermal emission of dust grains (Zhang \& Kwok 1992). This relatively large amount of dust may have some effects on the UV emission lines, especially the resonance lines. We, however, used a small amount for the dust to gas ratio, $\mathrm{M}_{\text {dust }} / \mathrm{M}_{\text {gas }}=0.001$, so the prediction for some of UV region resonance lines would not be expected to be good.

We did not employ a composite model geometry with an inhomogeneity in the nebular gas, 
but we constructed two models, 1) model A with $N_{H}=40000 \mathrm{~cm}^{-3}$, and 2) model B with a higher density in the shell, i.e. $N_{H}=80000 \mathrm{~cm}^{-3}$. The chemical abundances adopted in the above models are slightly different. Although model A and model B produce a fairly good fit to the lines, the former model has a serious problem with some of diagnostically important lines, e.g. [O II] 7321/7332. As indicated in Fig. 3, the higher density model would be desirable. Thus, we prefer the latter model with $N_{H}=80000 \mathrm{~cm}^{-3}$ (giving an electron density of $N_{\epsilon}=90000 \mathrm{~cm}^{-3}$ ), which gives a better prediction for most of lines, including the [O II] lines. Table 7 compares the observed and predicted intensities. Intensities from the ITS by AC79, and from our HES + IUE Archive data, are given in columns (3) and (4), respectively, while columns (5) and (6) list the predicted intensities. All of the values are on a scale of $\mathrm{I}(\mathrm{H} \beta)=100$.

The following discussion is based on model B. For most ions, fairly reasonable agreement between the observed and predicted intensities is achieved, but in some cases, especially [Ar V], we find a glaringly large discrepancy. The agreement for He I and He II seems fine. As usual, in predicting $\mathrm{He}$ lines, we corrected for collisionally excited contributions. The predictions for $\mathrm{C}$ seem fine, except for the recombination C II $\lambda 4267$ line, and C IV. In fact, Model A is favored by the observations of $\mathrm{C} \mathrm{IV}$. Predictions for the ions of $\mathrm{N}, \mathrm{O}, \mathrm{Ne}, \mathrm{S}$, and $\mathrm{Cl}$ seem generally successful. The [S II] $6717 / 6731$ lines have a problem. However, the agreement for other sulfur lines appears fine. Although the [S IV] IR line available from Beck et al. (1981) involves an uncertain extinction correction, we were also able to fit this line. Here, the lower density model A seems to fit the [S II] lines better. Rare elements like $\mathrm{Si}$ and $\mathrm{K}$ are all represented by single ionization stages: silicon by Si III], and potassium by [K IV]. Hence, agreement for these ions can be assured, and the abundances of these elements can be found by the model.

The electron temperatures indicated by the diagnostics are $\mathrm{T}_{\epsilon}=12000-14500 \mathrm{~K}, 11800 \mathrm{~K}$ and $11500 \mathrm{~K}$, for [O II], [O III], and [Ar III], respectively. However, model B predicts $\mathrm{T}_{\epsilon}=12500 \mathrm{~K}$, $11800 \mathrm{~K}$ and $12300 \mathrm{~K}$, respectively. Thus, the relatively higher temperatures indicated by the [O II] diagnostic, in contrast to the average electron temperature deduced from [O III], seem to be confirmed by the model. The model obviously cannot be consistent with the large scatter indicated by other diagnostics. There also appear to be some observational errors involved: for example, the diagnostics indicate a very low temperature for the $[\mathrm{N} \mathrm{II}]$ lines, but the model did not predict such a relatively low temperature, i.e. $\mathrm{T}_{\epsilon}<11000 \mathrm{~K}$ ([N II]: see Fig. 3 ) vs. $12600 \mathrm{~K}$ (model B). Since our model structures are basically simple homogeneous shells, they do not admit a point to point fluctuation of $N_{\epsilon}$. Note also that our photoionization models require a higher effective temperature for the CSPN, contrary to the derivations by Zhang \& Kwok (1992) or Köppen \& Tarafdar (1978). The CSPN temperature of IC 5117 is likely to be high, around $\mathrm{T}_{\text {eff }}=120000 \mathrm{~K}$.

We tried to simplify the problem by choosing a single diagnostic point to represent the physical conditions of PN. As a result, our model predicts the central star visual brightness as being one magnitude dimmer than observed, and, in addition, the predicted nebular size is slightly overestimated. This contradiction may disappear if we employ a WR-type model atmosphere for the CSPN (see Hyung et al. 2000). 


\section{Abundances}

\subsection{Ionic Concentrations}

With the appropriate electron temperature, $\mathrm{T}_{\epsilon}$, and electron density, $N_{\epsilon}$, we are now able to obtain the ionic concentrations by well-known formulae (see e.g. Aller 1984), updated with the most recent and reliable values of atomic constants. Table 8 presents the ionic concentration calculated from the interstellar extinction-corrected intensities, i.e. the UV region IUE and optical HES data listed in Tables 2 and 4. Successive columns present: ion involved, wavelength, intensity, and the values of $N($ ion $) / N\left(\mathrm{H}^{+}\right)$. In the last column, the summations of the ionic concentrations are obtained via weighting by the line intensities. In the case where the line measurements are uncertain or too weak, the results are discarded. For the choice of electron temperature and density in calculating each ionic concentration, we do not use all of the details discussed in Section 3. Instead, we applied a single representative diagnostic electron temperature and density to simplify the problem. As discussed earlier in Section 3 and in Fig. 3, there are two probable diagnostic points which may be suitable for our purpose, i.e. a) $N_{\epsilon} \sim 40000 \mathrm{~cm}^{-3}$ and $\mathrm{T}_{\epsilon} \sim 13000 \mathrm{~K}$; and b) $N_{\epsilon} \sim 90000 \mathrm{~cm}^{-3}$ and $\mathrm{T}_{\epsilon}=12000 \mathrm{~K}$. If we adopt the former as the physical state of IC 5117 , we find a large discordance in derived ionic concentrations in some ions. For example, the [O II] ionic concentration found by $\lambda 3727,3729$ is only $1 / 4$ times that by $\lambda 7320,7330$. However, if we adopt the latter higher density case as a physical condition, the disagreement becomes smaller (See Table 8). The physical condition of $N_{\epsilon}=90000 \mathrm{~cm}^{-3}$ and $\mathrm{T}_{\epsilon}=12000 \mathrm{~K}$, which has been discussed in previous sections, appears to be more appropriate than the other lower density.

We found the ionic concentrations for both He I and He II. The latter is about $10 \%$ of the former. The combined total is lower than the previous result found in the literature (see Section 5.2). For some ions such as carbon and silicon, the optical data are unavailable, forcing us to rely solely on the IUE measurements. Virtually all of the $\mathrm{C}$ ions are accounted for by $\left(\mathrm{C}^{+}, \mathrm{C}^{2+}, \mathrm{C}^{3+}\right)$, and in fact mainly by $\left(\mathrm{C}^{2+}\right)$; here, as usual, ionic concentrations of $\mathrm{C}^{+}, \mathrm{C}^{2+}$ and $\mathrm{C}^{3+}$ are derived from the UV lines, assumed to be collisionally excited. Similarly, the summation of oxygen ionic concentrations can be found from $\mathrm{O}^{+}$and $\mathrm{O}^{2+}$. The $\mathrm{O}^{2+}$ ionic concentration obtained from the IUE O III] $1661 / 1666$ is slightly higher than those from the optical [O III] lines. In this case, we ignored the IUE lines because of the relatively weak line intensities. Ionic concentrations for neon arc availablc for $\left(\mathrm{Nc}^{2+}, \mathrm{Nc}^{3+}\right)$ from the HES and IUE, respectively. The $\mathrm{Ne}^{3+}$ ionic concentration obtained from the weakly detected IUE line is about $25 \%$ of that found from the optical lines. For sulfur, we were able to calculate three ionic concentrations $\left(\mathrm{S}^{+}, \mathrm{S}^{2+}, \mathrm{S}^{3+}\right)$. The first two concentrations are measured from our HES data, while the $\mathrm{S}^{3+}$ concentration is based on the line intensity measured by Beck et al. (1981). Argon is mostly represented by $\mathrm{Ar}^{2+}$ and $\mathrm{Ar}^{3+}$, with a weak contribution from $\mathrm{Ar}^{4+}$. For chlorine, two ionic concentrations, $\mathrm{Cl}^{2+}$ and $\mathrm{Cl}^{3+}$, are available; but the theoretical model indicates a fairly large contribution, i.e. $\sim 50 \%$ from $\mathrm{Cl}^{4+}$ (see section 5.2). We are also able to find the ionic concentration for other rare elements. For example, potassium and silicon are represented by single ions, [K IV] and [Si III]. 


\subsection{Abundance Determinations}

To determine the abundance of IC 5117, we used two methods: 1) the Ionization Correction Factors (ICF's) method, coupled with the derivation of ionic concentrations as described in Section 5.1 , and 2) models. The latter method is to use the best model available, e.g. as described in Section 4, with adopted model abundances; while the former method calculates the fractional ionic concentration for each ionic stage, and uses the ICF's suggested by the model for the unobserved ionic stages.

The elemental abundances (relative to $N\left(\mathrm{H}^{+}\right)$of IC 5117 are given in Table 9. The second column of this table lists the $\Sigma N($ ion $) / N\left(\mathrm{H}^{+}\right)$from Table 8 , and the 3rd column lists the ICF obtained from the theoretical model in section 4 . The 4th column gives the ICF abundance, $N(\mathrm{ICF})$, obtained from the ionic concentration by applying the ICF's for the unobserved ionic stages, i.e. by multiplying the 2 nd column by the 3 rd one. The 5 th column gives the model abundances, $N$ (model), adopted from model B; the 6 th column gives the logarithmic difference, i.e. $\triangle=\log N(\mathrm{ICF})-\log N($ Model $)$, which is relatively small $(|\Delta|<<0.10)$ for most elements. The 7 th column gives the recommended abundance for IC 5117, while the 8th column lists the previous estimation for IC 5117 by Aller and Czyzak (1983). The last two columns list the 'average' PN abundance found by Aller and Czyzak (1983, AC83) and by Kingsburgh and Barlow (1984B), and the solar abundance by Grevesse and Noels (1993).

Both the ICF and model results are in good accord, and the abundances derived seem reasonable accurate. However, the current results are quite different from the AC79 result for C, $\mathrm{Ne}$, and K. Rudy et al. (2001) recently measured the near-infrared spectrum from 0.8 to $2.5 \mu \mathrm{m}$. They found $\mathrm{He} / \mathrm{H} \sim 0.113$, similar to the $\mathrm{AC} 83$ result, while our derivation produces a $10 \%$ lower value, at about the solar abundance, i.e. $\mathrm{He} / \mathrm{H} \sim 0.1$. This difference may be due to the density employed in the assumed physical conditions. To fit the helium lines with the lower gas density in the shell, we need to increase the helium abundance. Our result, which is based on a relatively good photoionization model, is perhaps more accurate than that from AC83, because of the higher quality data, and improved model.

For three elements, $\mathrm{C}, \mathrm{N}$ and $\mathrm{Ar}$, there are big discrepancies between the ICF and model results, $|\triangle| \sim 0.1$. The model cannot fit the $C$ IV lines, so we adopt the carbon abundance, close to the $\mathrm{ICF}$ method, i.e. $\mathrm{C} / \mathrm{H} \simeq 5.0(-4)$. Our derived $\mathrm{C} / \mathrm{H}$ value is lower than $\mathrm{AC} 83$ value by a factor of two, i.e. $8.9(-4)$ from AC83. Since the abundance derived here is for a gas phase, some carbon may be tied up in grains. Based on our derivation, we suggest that the carbon abundance is likely to be close to the solar but depleted relative to the average PN. Comparing our result with AC83 for other elements, we note that there exists a large discrepancy in $\mathrm{Ne}$ and $\mathrm{K}$. For other elements, our abundances are in accord with AC83: our derived abundances are slightly less than those of $\mathrm{AC} 83$ in $\mathrm{S}$ and $\mathrm{Ar}$, though the opposite is true for $\mathrm{Cl}$ and $\mathrm{K}$.

The current derivation of $\mathrm{N}$ and $\mathrm{O}$ abundances agree with those of $\mathrm{AC} 83$, i.e. $\mathrm{N} / \mathrm{H} \sim 1.3(-$ 4) and $\mathrm{O} / \mathrm{H} \sim 3.8(-4)$. Thus, these are close to the average PN, but less abundant than solar. 
Similarly, both $\mathrm{Ne}$ and $\mathrm{Ar}$ abundances appear close to the average $\mathrm{PN}$ value. We obtained $\mathrm{Ne} / \mathrm{H} \sim$ $6.5(-5)$, vs. 9.3(-5) from $\mathrm{AC} 83$. We derived the sulfur abundance, $\mathrm{S} / \mathrm{H} \sim 8.9(-6), 25 \%$ lower than in AC83. Rudy et al. (2001) found an even lower value, 7.5(-6). We derived a chlorine abundance of $1.85(-7), 30 \%$ higher than AC83 value, which is slightly less abundant than the average PN, but lower than solar.

\section{Conclusion}

We constructed a nebular model for IC 5117 based on the diagnostic information, and found the abundances in this PN. These were compared with those from AC83, and also with the average $\mathrm{PN}$. For He, C, and $\mathrm{K}$, we found a factor of 2 difference from AC83. We believe our result is substantially improved over the previous determination by $\mathrm{AC} 83$, due to higher quality data and a better theoretical model employed. Our study suggests the $\mathrm{C} / \mathrm{H}, \mathrm{Ne} / \mathrm{H}$, and $\mathrm{Ar} / \mathrm{H}$ ratios are lower than the average PN. However, we found no evidence of metal deficiency in other elements: the $\mathrm{He} / \mathrm{H}, \mathrm{N} / \mathrm{H}, \mathrm{O} / \mathrm{H}$, and $\mathrm{Cl} / \mathrm{H}$ ratios are close to the average $\mathrm{PN}$. Only Si involves a large ICF.

If the assumed distance to the PN IC 5117 is correct, the employed CSPN temperature and luminosity should give us a CSPN mass. Taking $\mathrm{L}(\star)$ and $T(\star)$ at their face values (see Table 6 ) and utilizing Schönberner's (1983) evolutionary tracks, we derive a CSPN mass of about $0.60 \mathrm{M}_{\odot}$. In addition, these tracts suggest a corresponding age of about 7000 years, as evolved from an AGB progenitor. The AGB must have been a C-rich star, i.e. C/O ratio greater than 1. The central star must have been slightly more massive than our Sun on its main sequence phase. CO emission is commonly observed in PNe of Peimbert's Type 1 or Greig's Class B (Greig 1972; Huggins \& Healy 1989). However, the chemical abundances in IC 5117 is fairly normal, certainly not expected from a Peimbert type I PN. The mass of the CSPN of IC 5117 is too low for a Peimbert's Type I PN.

The VLA-6 cm continuum observation by Kwok (1995) shows that IC 5117 has a smooth brightness distribution, and its is slightly extended in the $\mathrm{E}-\mathrm{W}$ direction. Although the structure of IC 5117 is unresolved, and it may be bipolar in the first approximation (Rudy et al. 2001), it may, in fact, have a much more complicated morphology: the diagnostics indicate that the nebula is inhomogeneous, with perhaps as high density as $N_{\epsilon}=100000 \mathrm{~cm}^{-3}$ and perhaps as low as $30000-$ $40000 \mathrm{~cm}^{-3}$. However, our model and diagnostic analyses show the nebular physical condition can be successfully represented by a homogeneous shell with $N_{H}=80000 \mathrm{~cm}^{-3}$. The model predictions are in general successful. The electron temperatures indicated by the diagnostics are relatively high, and this can be modeled with a CSPN $T_{\text {eff }}$ of about $120000 \mathrm{~K}$. Diagnostics and models suggest a relatively high excitation, and the models also predict higher excitation temperatures, e.g. in [O II], [O III], and [Ar III]. Since detailed images are not available, constructing a sophisticated composite model would be too hasty at this stage. However, one must certainly introduce a refinement in the theoretical model construction, as soon as the high spatial resolution (sub-arc-second scale) imaging becomes available in the future. 
We express our gratitude to the technical staff of Lick Observatory, who helped us secure these data. We also acknowledge Dr. André B. Fletcher for his careful review of this paper; and Dr. Lauren Likkel for her measurements of all the near-UV lines from the green tube ITS data. This research was supported by the KRF grant No. 2000-015-DP0445, by the Korea MOST Grant No. Star 01-2-500-00, by the KOSEF Grant No. 2000-1-113-001-5, and by STScI Grant No. AR 06372-01 95A.

\section{REFERENCES}

Aaquist, O. B., \& Kwok, S. 1990, A\&AS, 84, 229

Acker, A., Ochsenbein, F., Stenholm, B., Tylenda, R., Marcout, J., \& Schohn, C., 1992, Strasbourg -ESO Catalogue of Galactic Planetary Nebulae, Garchung bei Munchen, European Southern Observatory

Aller, L. H., 1984, Physics of Thermal Gaseous Nebulae, (Dordrecht: Reidel Publishing Company)

Aller, L. H., \& Czyzak, S. J. 1983, ApJS, 51, 211 (AC83)

Aller, L. H., \& Czyzak, S. J. 1979, Ap\&SS, 1979, 62, 397 (AC79)

Aller, L. H., \& Liller W., 1968, in Middlehurst B.M. and Aller, L.H., eds, Nebulae and Interstellar Matter, University of Chicago Press, Chicago, ch. 9, p 498

Beck, S.C. Lacy, J.H., Townes, C.H., Aller, L.H., Gerballe, T.R. \& Baas, F. 1981, ApJ, 249, 592

Greig, W. E. 1972 A\&A, 18, 70

Gussie, G. T., \& Taylor, A. R. 1995, MNRAS, 273, 801

Grevesse, N., \& Noels, A. 1993, in Origin and Evolution of Elements, eds. Prantzos, N., VangioniFlam, E. Casse, M. (Cambridge Univ. Press, Cambridge, U.K.), 15

Herrick, S., 1935, Lick Obs. Bull., 17, 85

Hubeny, I. 1988, Computer Phys. Comm., 52, 103

Huggins P. J., \& Healy, A. P. 1989, ApJ, 346, 201

Hyung, S. 1994, ApJS, 90, 119

Hyung, S., \& Aller, L. H., 1996, MNRAS, 278, 551

Hyung, S., Aller, L. H., Feibelman, W. A., \& Lee, W.-B. 2001, AJ, in press

Hyung, S., Aller, L. H., Feibelman, W. A., Lee, W.-B., \& de Koter, A. 2000, MNRAS, 318, 77 
Keenan, F. P., Aller, L. H., Ramsbottom, C. A., Bell, K. L., Crawford, F. L., \& Hyung, S. 2000, Proc. Natl. Acad Sci. USA, 97, 4551

Keenan, F. P., Aller, L. H., Bell, K. L., Crawford, F. L., Feibelman, W. A., Hyung, S., McKenna, F. C., \& McLaughlin, B. M. 1999, MNRAS, 304, 27

Keenan, F. P., McKenna, F. C., Bell, K. L., Ramsbottom, C. A., Wickstead, A. W., Aller, L. H., \& Hyung, S. 1997, ApJ, 487, 457

Keenan, F. P., Aller, L. H., Bell, K. L., Hyung, S., McKenna, F. C., \& Ramsbottom, C. A. 1996, MNRAS, 281, 1073

Köppen, J., \& Tarafdar, S. P., 1978, A\&A, 69, 363

Kingsburgh, R. L., \& Barlow, M., J., 1994, MNRAS, 271, 257

Kwok, S. 1985, AJ, 90, 49

Likkel, L., \& Aller, L. H. 1986, ApJ, 301, 825 (LA86)

Mamon, G. A., Glassgold, A. E., \& Huggins, P. J. 1988, ApJ, 328, 797

Miranda, L. F., Torrelles, J. M., \& Eiroa, C. 1995, ApJ, 446, L39

Moore, C. E. 1993, in J. W. Gallagher, ed., 'Tables of Spectra of H, C, N, and O, atoms and ions', CRC press, London

Moore, C. E. 1974, A Multiplet Table of Astrophysical Interest, National Bureau of Standards, No.40

Peimbert, M., Luridiana, V., \& Torres-Peimbert, S. 1995, RMxA\&A, 31, 147

Rudy, R. J., Lynch, D. K., Mazuk, S., Puertter, R. C., \& Dearborn, D. S. P. 2001, AJ, 121, 362

Seaton, M. J. 1979, MNRAS, 187, 73P

Schönberner, B. 1983, ApJ, 272, 708

Zhang, C. Y. 1995, ApJS, 98, 659

Zhang, C. Y., \& Kwok, S. 1992, ApJ, 385, 255 


\section{Calibrated Flux}

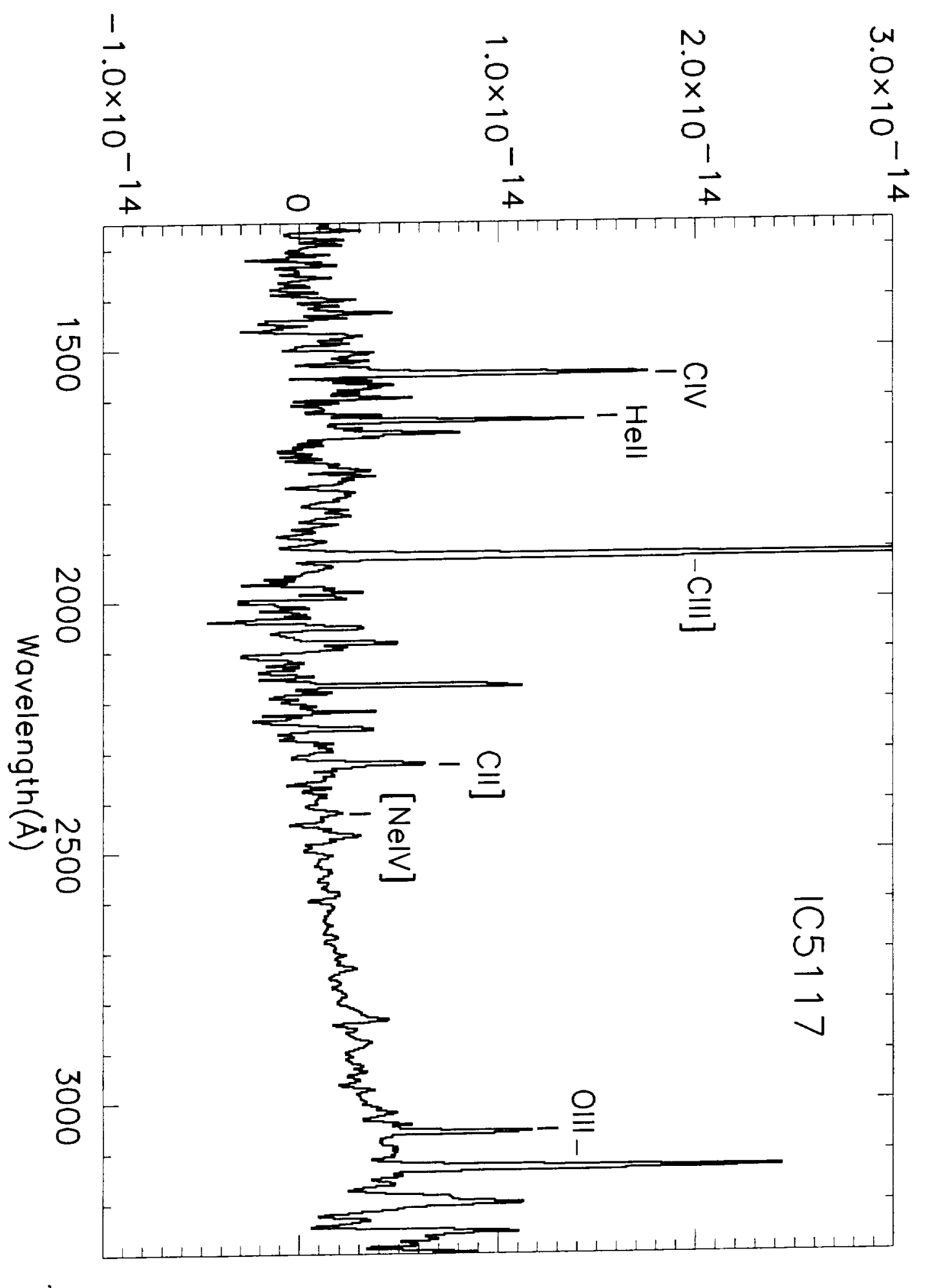

Fig. 1.- The Ultraviolet Spectrum of IC 5117 (extinction correction not applied in this diagram). Although the baseline is below the zero, the flux measurement can be done, correctly (flux unit is erg $\mathrm{cm}^{-2} \mathrm{~s}^{-1} \AA^{-1}$ ). Plot was smoothed by a three point running average. 
Fig. 1. - The Ultraviolet Spectrum of IC 5117 (extinction correction not applied in this diagram). Although the baseline is below the zero, the flux measurement can be done, correctly (flux unit is erg $\mathrm{cm}^{-2} \mathrm{~s}^{-1} \AA^{-1}$ ). Plot was smoothed by a three point running average. 

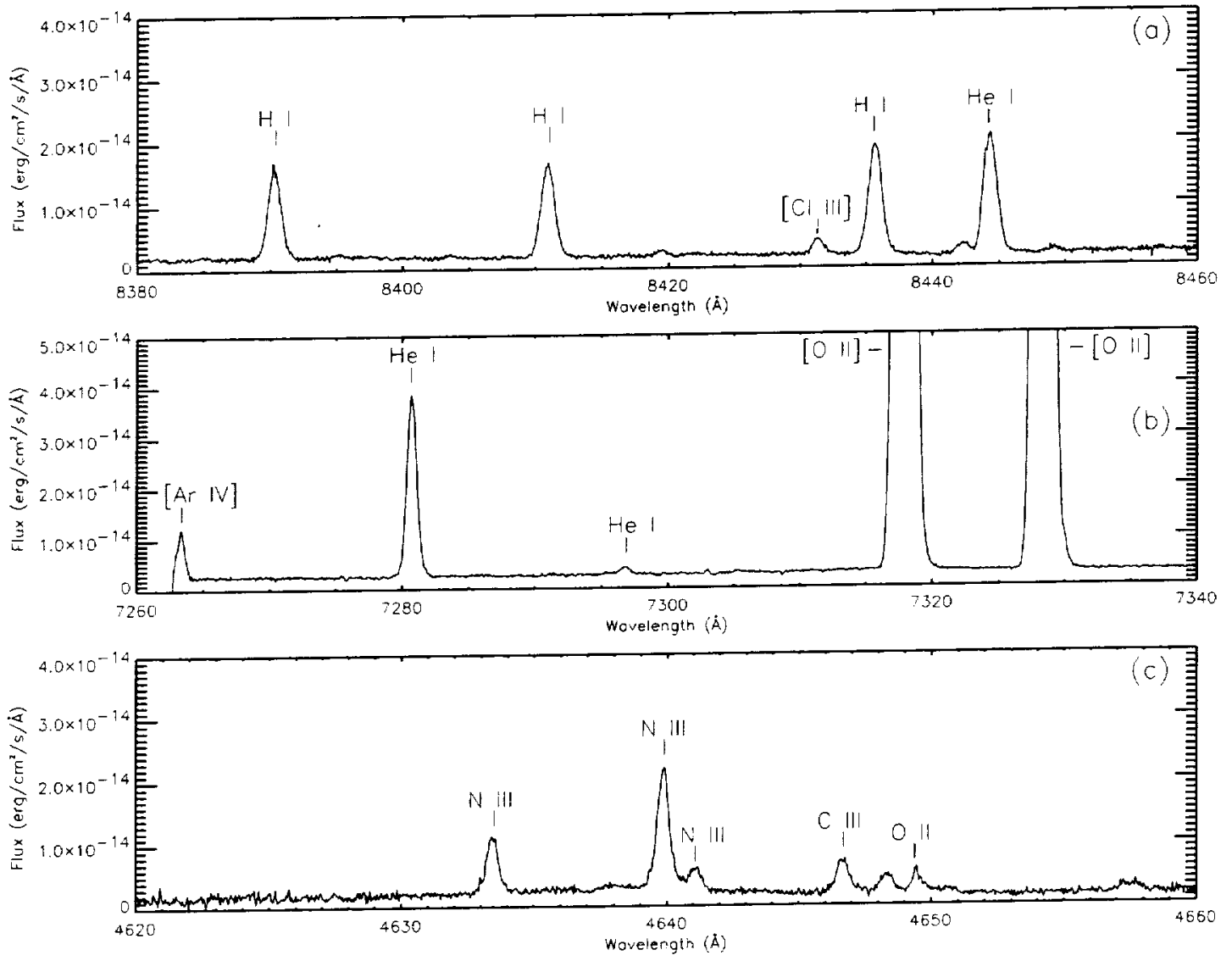

Fig. 2.- Plot of the HES spectrum of IC 5117 - (a) $8380-8460 \AA$, (b) $7260-7340 \AA$, (c) $4620-$ $4660 \AA$. Radial velocity and interstellar extinction corrections are not applied to this spectral scan. 


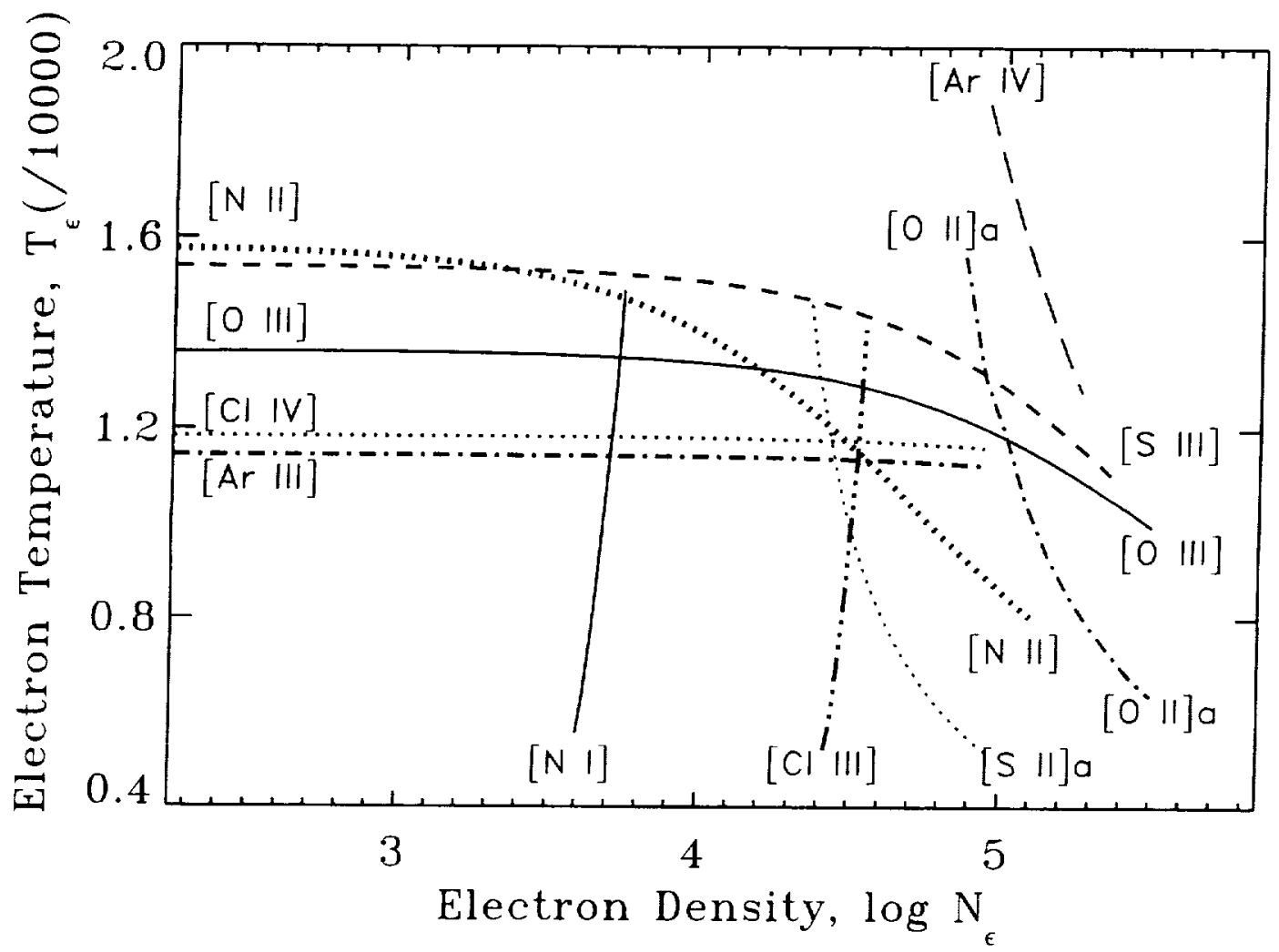

Fig. 3.- Diagnostic diagram for IC $5117-\mathrm{T}_{\epsilon}$ vs. $\log N_{\epsilon}$. Here [O II]a and [S II]a refer to the transauroral to the nebular transition. These diagnostics are from lines involving $\mathrm{p}^{2}$ and $\mathrm{p}^{4}$ electrons. See Table 5 and text for additional diagnostics involving $\mathrm{p}^{3}$ electrons. 
Table 1. Some basic data for IC 5117 (PN G 089.8-05.1).

Basic Data

$\alpha=21^{h} 32^{m} 30^{s} .9, \delta=44^{\circ} 35^{\prime} 47^{\prime \prime}(2000)$,

Diameter $\simeq 1.2^{\prime \prime}-1.5^{\prime \prime} ; \log \mathrm{F}(\mathrm{H} \beta)=-11.37 \pm 0.01\left[\mathrm{erg} \mathrm{cm}^{-2} \mathrm{~s}^{-1}\right]$

Excitation class: 6.0

Radial Velocity $=-26.1 \pm 1.3 \mathrm{~km} \mathrm{~s}^{-1} ;-38.69 \pm 0.70 \mathrm{~km} \mathrm{~s}^{-1}$ (this paper)

Expansion velocity $=16.5 \& 21.5 \mathrm{~km} \mathrm{~s}^{-1}$ ([O III] \& [N II], respectively)

Central star: $\mathrm{m}_{B}=17.5, \mathrm{~m}_{V}=16.7, \mathrm{WR}$

$\mathrm{T}(\star)=120000 \mathrm{~K}$ (this paper)

References. - These data are from Acker et al. (1992), unless otherwise indicated. 
Table 2. IUE spectral line intensity.

\begin{tabular}{llcccc}
\hline \hline$\lambda$ (obs) & $\lambda$ (rest) & Ion & $k_{\lambda}{ }^{a}$ & Intensity & Flux \\
\hline 1251.89 & $1238 / 40$ & N v & 1.606 & 33 & $1.2:$ \\
1426.54 & 1440.36 & C IV? & 1.283 & 30 & 2.8 \\
1548.91 & $1548 / 50$ & C IV & 1.184 & 127 & 16.1 \\
1573.76 & 1574.80 & [Ne v] & 1.168 & 23 & 3.0 \\
1596.42 & 1596.37 & C IV? & 1.156 & 16 & 2.2 \\
1641.08 & 1640.39 & He II & 1.136 & 59 & 8.6 \\
1665.88 & $1661 / 66$ & O III] & 1.128 & 38 & 5.7 \\
1715.52 & 1718 & N IV & 1.119 & 15 & 2.3 \\
1751.66 & $1746 / 70$ & N III] & 1.120 & 12 & 1.9 \\
1880.82 & $1882 / 92$ & Si III] & 1.193 & 8.1 & 1.0 \\
1908.88 & $1907 / 09$ & C III] & 1.228 & 418 & 46.4 \\
2323.52 & $2325 / 29$ & C II] & 1.360 & 83 & 6.2 \\
2340.85 & $2334-50$ & Si II] & 1.315 & 41 & 3.5 \\
2385.21 & 2385 & He II & 1.204 & 23 & 2.8 \\
2421.56 & $2422 / 24$ & [Ne IV] & 1.121 & 11 & 1.7 \\
2466.76 & 2470.33 & [O II] & 1.031 & 13 & 2.6 \\
2832.52 & 2829 & He I & 0.622 & 5.8 & 3.9 \\
3022.15 & 3023 & O III & 0.507 & 4.1 & 3.9 \\
3059.09 & 3047.13 & O III & 0.489 & 5.6 & 5.6 \\
3066.78 & $063 / 71$ & [N II] & 0.485 & 4.7 & 4.8 \\
3129.78 & 3232.90 & O III & 0.456 & 9.5 & 10.5 \\
3199.40 & 1640.39 & He II & 0.426 & 6.1 & 7.3 \\
\hline & & & & &
\end{tabular}

${ }^{a}$ the extinction parameter according to Seaton (1979)

Note. - The UV fluxes in col. (6) are line intensities in units of $10^{-14} \mathrm{erg} \mathrm{cm} \mathrm{cm}^{-2} \mathrm{~s}^{-1} \AA^{-1}$, and the intensities in col. (5) are given based on the scale of $\mathrm{I}(\mathrm{H} \beta)=100$; the interstellar extinction corrections are made assuming $C=1.29$ [or $\mathrm{E}(\mathrm{B}-\mathrm{V})=0.88]$. Colon for fluxes means the estimated error is large, $\pm 40 \%$, while others are $\pm 15 \%$. Only IUE Spectra SWP 31825 (150min, 1987 Sep 11) and LWP 05884 (295min, 1985 May 2) were measured in deriving these results. 
Table 3. Image tube scanner observation of IC 5117.

\begin{tabular}{cccccc}
\hline \hline$\lambda$ (obs) & Ion & $k_{\lambda}$ & Intensity & Flux & Remarks \\
\hline 3299.00 & O III & 0.387 & 0.548 & 0.74 & B \\
3312.00 & O III & 0.383 & 1.08 & 1.48 & B \\
3317.36 & line? & 0.381 & 1.24 & 1.71 & \\
3341.00 & O III & 0.372 & 1.81 & 2.56 & B \\
3428.00 & O III & 0.343 & 0.669 & 1.03 & B \\
3444.00 & O III & 0.338 & 3.22 & 5.04 & B \\
3512.00 & He I & 0.317 & 0.145 & 0.24 & \\
3530.00 & He I & 0.311 & 0.123 & 0.21 & \\
3554.00 & He I & 0.304 & 0.278 & 0.48 & \\
3587.00 & He I & 0.295 & 0.300 & 0.53 & \\
3613.00 & He I & 0.288 & 0.148 & 0.27 & \\
3634.00 & He I & 0.282 & 0.340 & 0.63 & \\
3679.99 & H I & 0.270 & 0.103 & 0.20 & \\
3683.38 & H I & 0.269 & 0.167 & 0.32 & \\
3687.39 & H I & 0.268 & 0.247 & 0.48 & \\
3692.23 & H I & 0.266 & 0.388 & 0.75 & \\
3697.96 & H I & 0.265 & 0.500 & 0.97 & \\
3727.00 & [O II] & 0.265 & 14.7 & 28.5 & \\
3871.70 & H I & 0.227 & 95.2 & 207 & \\
\hline
\end{tabular}

Note. - ITS measurements by Likkel: 'B' in the remarks column, show lines from the Bowen fluorescent mechanism (see Likkel \& Aller 1986, LA86). Interstellar extinction corrected intensities are given on the scale of $\mathrm{I}(\mathrm{H} \beta)=100(C=1.29)$, while fluxes are given in units of

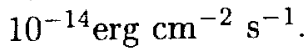


Table 4. Optical Spectrum of IC 5117.

\begin{tabular}{|c|c|c|c|c|c|c|c|}
\hline$\lambda(\mathrm{obs})$ & $\lambda($ rest $)$ & Ion & Mult. & $k_{\lambda}$ & Int(HES) & Flux(HES) & RMS \\
\hline 3703.81 & 3703.86 & H I & $\mathrm{H} 16$ & 0.272 & 1.304 & 0.54 & $26 \%$ \\
\hline \multirow[t]{2}{*}{3712.04} & .3711 .97 & $\mathrm{H} \mathrm{I}$ & $\mathrm{H} 15$ & 0.269 & 1.557 & 0.65 & $39 \%$ \\
\hline & 3721.94 & $\mathrm{H} \mathrm{I}$ & $\mathrm{H} 14$ & & & & \\
\hline 3721.87 & 3721.83 & [S III] & $(2 F)$ & 0.267 & 2.694 & 1.14 & $6 \%$ \\
\hline 3726.03 & 3726.03 & {$[\mathrm{O}$ II $]$} & $(1 F)$ & 0.266 & 8.666 & 3.68 & $2 \%$ \\
\hline 3728.75 & 3728.82 & {$[\mathrm{O} \mathrm{II}]$} & $(1 F)$ & 0.265 & 2.798 & 1.19 & $4 \%$ \\
\hline 3734.39 & 3734.37 & $\mathrm{H} \mathrm{I}$ & $\mathrm{H} 13$ & 0.263 & 2.084 & 0.89 & \\
\hline 3750.16 & 3750.15 & $\mathrm{H} \mathrm{I}$ & $\mathrm{H} 12$ & 0.259 & 2.570 & 1.11 & $6 \%$ \\
\hline 3754.73 & 3754.67 & $\mathrm{O}$ III & (2) & 0.258 & 0.375 & 0.16 & $7 \%$ \\
\hline \multirow[t]{2}{*}{3759.92} & 3759.81 & O III & $(2)$ & 0.256 & 0.991 & 0.43 & $11 \%$ \\
\hline & 3771.08 & $\mathrm{~N}$ III & (4) & & & & \\
\hline 3770.85 & 3770.63 & $\mathrm{H} \mathrm{I}$ & $\mathrm{H} 11$ & 0.253 & 3.300 & 1.46 & $18 \%$ \\
\hline 3797.90 & 3797.90 & $\mathrm{H} \mathrm{I}$ & $\mathrm{H} 10$ & 0.246 & 4.110 & 1.86 & $4 \%$ \\
\hline 3819.63 & 3819.61 & $\mathrm{He} I$ & $(22)$ & 0.241 & 0.822 & 0.38 & $18 \%$ \\
\hline 3835.40 & 3835.39 & $\mathrm{H} \mathrm{I}$ & H9 & 0.236 & 5.650 & 2.64 & $22 \%$ \\
\hline 3858.99 & 3858.07 & $\mathrm{He} \mathrm{II}^{\#}$ & $4-17$ & 0.230 & 0.226 & 0.11 & $51 \%$ \\
\hline 3868.91 & 3868.71 & [Ne III] & $(1 F)$ & 0.228 & 103.8 & 49.77 & $7 \%$ \\
\hline 3888.96 & 3889.05 & $\mathrm{H} \mathrm{I}$ & $\mathrm{H} 8$ & 0.223 & 11.48 & 5.60 & $11 \%$ \\
\hline 3964.75 & 3964.73 & $\mathrm{He} \mathrm{I}$ & (5) & 0.204 & 0.604 & 0.31 & \\
\hline 3967.49 & 3967.41 & [Ne III] & $(1 F)$ & 0.203 & 42.64 & 22.13 & $10 \%$ \\
\hline 3970.09 & 3970.07 & $\mathrm{H} \mathrm{I}$ & $\mathrm{H} \epsilon$ & 0.203 & 13.30 & 6.92 & $23 \%$ \\
\hline 4009.43 & 4009.27 & He I & $(55)$ & 0.193 & 0.215 & 0.12 & $2 \%$ \\
\hline 4026.16 & 4026.36 & $\mathrm{He} \mathrm{I}$ & $(18)$ & 0.189 & 2.214 & 1.20 & $2 \%$ \\
\hline 4047.09 & 4047.80 & $\mathrm{O}_{\mathrm{II}}^{\#}$ & & 0.185 & 0.292 & 0.16 & \\
\hline 4068.63 & 4068.60 & {$[\mathrm{~S} \mathrm{III}]$} & $(1 \mathrm{~F})$ & 0.180 & 3.030 & 1.70 & $4 \%$ \\
\hline 4076.29 & 4076.35 & {$[\mathrm{~S} \mathrm{II}]$} & $(1 F)$ & 0.178 & 1.130 & 0.64 & $2 \%$ \\
\hline 4097.38 & 4097.31 & $\mathrm{~N}$ III & (1) & 0.173 & 1.102 & 0.63 & $5 \%$ \\
\hline 4099.97 & 4100.04 & He II & $(4-12)$ & 0.172 & 0.134 & 0.08 & \\
\hline 4101.78 & 4101.76 & $\mathrm{H} \mathrm{I}$ & $\mathrm{H} \delta$ & 0.172 & 25.70 & 14.76 & $3 \%$ \\
\hline \multirow[t]{2}{*}{4103.42} & 4103.37 & $\mathrm{~N}$ III & (1) & 0.172 & 0.502 & 0.29 & $7 \%$ \\
\hline & 4121.46 & O II & (19) & & & & \\
\hline \multirow[t]{2}{*}{4120.93} & 4120.81 & $\mathrm{He} \mathrm{I}$ & $(16)$ & 0.168 & 0.280 & 0.16 & $35 \%$ \\
\hline & 4143.77 & O II & $(106)$ & & & & \\
\hline
\end{tabular}


Table 4-Continued

\begin{tabular}{|c|c|c|c|c|c|c|c|}
\hline$\lambda(\mathrm{obs})$ & $\lambda($ rest $)$ & Ion & Mult. & $k_{\lambda}$ & $\operatorname{Int}(\mathrm{HES})$ & Flux(HES) & RMS \\
\hline 4143.72 & 4143.76 & $\mathrm{He} \mathrm{I}$ & $(53)$ & 0.163 & 0.287 & 0.17 & $6 \%$ \\
\hline 4161.97 & 4162.86 & $\mathrm{C}$ III & (21) & 0.159 & 0.165 & 0.10 & $10 \%$ \\
\hline 4187.00 & 4186.90 & C III & (18) & 0.154 & 0.196 & 0.12 & $13 \%$ \\
\hline 4199.93 & 4199.83 & He II & $(4-11)$ & 0.152 & 0.222 & 0.14 & $11 \%$ \\
\hline 4267.14 & 4267.18 & $\mathrm{C}$ II & $(6)$ & 0.141 & 0.483 & 0.31 & $12 \%$ \\
\hline 4338.70 & 4338.67 & He II & $(4-10)$ & 0.129 & 0.308 & 0.20 & $17 \%$ \\
\hline 4340.61 & 4340.47 & $\mathrm{H} \mathrm{I}$ & $\mathrm{H} \gamma$ & 0.129 & 46.50 & 30.69 & $5 \%$ \\
\hline 4363.20 & 4363.21 & [O III] & $(2 F)$ & 0.124 & 19.72 & 13.24 & $5 \%$ \\
\hline 4373.83 & 4371.59 & $\mathrm{C} \mathrm{II}^{\#}$ & $(45)$ & 0.121 & 0.537 & 0.36 & $11 \%$ \\
\hline 4387.91 & 4387.93 & He I & (51) & 0.117 & 0.580 & 0.40 & $4 \%$ \\
\hline 4437.49 & 4437.55 & $\mathrm{He} \mathrm{I}$ & $(50)$ & 0.104 & 0.123 & 0.09 & $1 \%$ \\
\hline 4471.48 & 4471.48 & He I & (14) & 0.095 & 5.295 & 3.90 & $9 \%$ \\
\hline 4541.61 & 4541.59 & He II & (9) & 0.077 & 0.404 & 0.31 & $4 \%$ \\
\hline 4570.98 & 4571.00 & $\mathrm{Mg} \mathrm{I}]$ & (1) & 0.070 & 0.504 & 0.40 & $8 \%$ \\
\hline 4634.13 & 4634.16 & $\mathrm{~N}$ III & (2) & 0.054 & 0.608 & 0.51 & $8 \%$ \\
\hline 4640.56 & 4640.64 & $\mathrm{~N}$ III & (2) & 0.053 & 1.282 & 1.08 & $5 \%$ \\
\hline 4641.82 & 4641.81 & $\mathrm{~N}$ III & $(2)$ & 0.053 & 0.183 & 0.15 & \\
\hline 4647.40 & 4647.40 & $\mathrm{C}$ III & (1) & 0.051 & 0.436 & 0.37 & \\
\hline 4649.05 & 4649.14 & $\mathrm{O}$ II & (1) & 0.051 & 0.242 & 0.21 & $2 \%$ \\
\hline \multirow[t]{2}{*}{4650.23} & 4650.16 & C III & (1) & 0.050 & 0.219 & 0.19 & $5 \%$ \\
\hline & 4658.64 & $\mathrm{C} \mathrm{IV}$ & $(8)$ & & & & \\
\hline 4658.21 & 4658.10 & {$[\mathrm{Fe}$ III] } & $(3 F)$ & 0.049 & 0.152 & 0.13 & $5 \%$ \\
\hline 4685.71 & 4685.68 & He II & $(3-4)$ & 0.042 & 9.439 & 8.24 & $3 \%$ \\
\hline 4711.34 & 4711.34 & [Ar IV] & $(1 F)$ & 0.036 & 1.129 & 1.00 & $15 \%$ \\
\hline 4713.11 & 4713.14 & He I & $(12)$ & 0.036 & 0.778 & 0.69 & $17 \%$ \\
\hline 4725.58 & 4725.62 & {$[\mathrm{Ne}$ IV $]$} & $(1 F)$ & 0.033 & 0.108 & 0.10 & $14 \%$ \\
\hline 4740.40 & 4740.20 & {$[$ Ar IV] } & (1F) & 0.029 & 4.722 & 4.30 & $11 \%$ \\
\hline 4789.49 & 4789.45 & {$\left[\begin{array}{ll}F & \mathrm{II}\end{array}\right]$ ? } & & 0.018 & 0.040 & 0.04 & \\
\hline 4811.79 & & line? & & 0.012 & 0.056 & 0.05 & $15 \%$ \\
\hline 4859.45 & 4859.32 & He II & $(4-8)$ & 0.000 & 0.436 & 0.44 & $8 \%$ \\
\hline 4861.51 & 4861.33 & $\mathrm{H} \mathrm{I}$ & $\mathrm{H} \beta$ & 0.000 & 100.00 & 100.00 & $5 \%$ \\
\hline 4880.94 & 4881.11 & {$[\mathrm{Fe} \quad \mathrm{III}]$} & $(2 \mathrm{~F})$ & -0.005 & 0.071 & 0.07 & \\
\hline 4921.80 & 4921.93 & $\mathrm{He} I$ & (48) & -0.014 & 0.798 & 0.84 & $7 \%$ \\
\hline
\end{tabular}


Table 4-Continued

\begin{tabular}{|c|c|c|c|c|c|c|c|}
\hline$\lambda(\mathrm{obs})$ & $\lambda($ rest $)$ & Ion & Mult. & $k_{\lambda}$ & $\operatorname{Int}(\mathrm{HES})$ & Flux (HES) & $\mathrm{RMS}$ \\
\hline 4931.05 & 4931.30 & {$[\mathrm{O} \mathrm{III}]$} & $(1 F)$ & -0.017 & 0.166 & 0.18 & $4 \%$ \\
\hline 4948.71 & 4948.54 & [Fe III] & & -0.021 & 0.159 & 0.17 & $2 \%$ \\
\hline 4958.89 & 4958.92 & [O III] & $(1 F)$ & -0.023 & 395.0 & 425.6 & $3 \%$ \\
\hline 4996.23 & 4996.29 & $\dagger$ & & -0.032 & 0.449 & 0.50 & $15 \%$ \\
\hline 5007.11 & 5006.84 & {$[\mathrm{O} \mathrm{III}]$} & $(1 F)$ & -0.034 & 1346.4 & 1503.1 & $2 \%$ \\
\hline 5041.02 & 5041.06 & Si II & (5) & -0.041 & 0.207 & 0.24 & $18 \%$ \\
\hline \multirow[t]{2}{*}{5047.62} & 5047.74 & $\mathrm{He} \mathrm{I}$ & $(47)$ & -0.043 & 0.095 & 0.11 & $10 \%$ \\
\hline & 5056.35 & Si II & (5) & & & & \\
\hline \multirow[t]{2}{*}{5056.11} & 5056.02 & Si II & (5) & -0.045 & 0.194 & 0.22 & $6 \%$ \\
\hline & 5121.82 & $\mathrm{C}_{\text {II }}$ & (2) & & & & \\
\hline 5121.42 & 5121.69 & $\mathrm{C}$ II & $(12)$ & -0.058 & 0.039 & 0.05 & $14 \%$ \\
\hline \multirow[t]{2}{*}{5131.02} & 5131.41 & $\mathrm{C}$ III & $5 g-7 h$ & -0.060 & 0.080 & 0.10 & $10 \%$ \\
\hline & 5146.06 & $\mathrm{O}$ ? & $(28)$ & & & & \\
\hline 5145.63 & 5145.77 & {$[\mathrm{Fe} \quad \mathrm{VI}]$} & $(2 F)$ & -0.063 & 0.024 & 0.03 & $7 \%$ \\
\hline 5176.48 & 5176.40 & {$[\mathrm{Fe} \quad \mathrm{VI}]$} & $(2 F)$ & -0.070 & 0.025 & 0.03 & $23 \%$ \\
\hline 5191.52 & 5191.80 & [Ar III] & $(3 F)$ & -0.073 & 0.156 & 0.20 & $4 \%$ \\
\hline 5197.66 & 5197.90 & {$\left[\begin{array}{ll}\mathrm{N} & \mathrm{I}\end{array}\right]$} & $(1 F)$ & -0.074 & 0.151 & 0.19 & \\
\hline 5200.03 & 5200.26 & {$[\mathrm{~N} \mathrm{I}]$} & $(1 F)$ & -0.074 & 0.088 & 0.11 & $2 \%$ \\
\hline 5270.46 & 5270.40 & {$[\mathrm{Fe}$ III $]$} & $(1 F)$ & -0.089 & 0.084 & 0.11 & $11 \%$ \\
\hline 5323.23 & 5323.30 & {$[\mathrm{Cl}$ IV $]$} & $(3 F)$ & -0.100 & 0.044 & 0.06 & $1 \%$ \\
\hline 5342.10 & 5342.56 & $\mathrm{C}$ II & & -0.104 & 0.046 & 0.06 & $12 \%$ \\
\hline \multirow[t]{2}{*}{5345.77} & 5345.90 & {$[K \quad I V]$} & $(1 F)$ & -0.105 & 0.129 & 0.18 & $8 \%$ \\
\hline & 5412.00 & [Fe III] & $(1 F)$ & & & & \\
\hline 5411.51 & 5411.52 & He II & (2) $4-7$ & -0.118 & 0.922 & 1.35 & $1 \%$ \\
\hline 5461.84 & 5462.62 & $\mathrm{~N}$ II & $(29)$ & -0.128 & 0.215 & 0.33 & $12 \%$ \\
\hline 5517.65 & 5517.71 & {$[\mathrm{Cl} \mathrm{III}]$} & $(1 F)^{\circ}$ & -0.139 & 0.142 & 0.22 & $11 \%$ \\
\hline 5537.61 & 5537.88 & {$[\mathrm{Cl} \mathrm{III}]$} & $(1 F)$ & -0.143 & 0.433 & 0.69 & \\
\hline 5577.52 & 5577.34 & {$[\mathrm{O} \mathrm{I}]$} & $(3 F)$ & -0.152 & 0.139 & 0.23 & $8 \%$ \\
\hline 5592.07 & 5592.37 & O III & (5) & -0.155 & 0.036 & 0.06 & \\
\hline 5613.50 & 5614.7 & {$[\mathrm{Ca} \text { viI }]^{\#}$} & $?$ & -0.160 & 0.021 & 0.04 & $4 \%$ \\
\hline 5631.06 & 5631.07 & {$[\mathrm{Fe} \mathrm{vI}]$} & & -0.164 & 0.018 & 0.03 & $11 \%$ \\
\hline 5659.98 & 5660.20 & $\mathrm{C} \mathrm{IV}$ & (1) & -0.170 & 0.014 & 0.02 & $7 \%$ \\
\hline 5666.37 & 5666.64 & $\mathrm{~N}$ II & (3) & -0.172 & 0.014 & 0.03 & $3 \%$ \\
\hline
\end{tabular}


Table 4-Continued

\begin{tabular}{rrcccccc}
\hline \hline$\lambda$ (obs) & $\lambda$ (rest) & Ion & Mult. & $k_{\lambda}$ & Int(HES) & Flux(HES) & RMS \\
\hline 5680.13 & 5679.56 & N II & $(3)$ & -0.175 & 0.038 & 0.07 & $30 \%$ \\
5708.64 & 5710.76 & N II & $(3)$ & -0.181 & 0.026 & 0.05 & $28 \%$ \\
5754.55 & 5754.64 & [N II $]$ & $(3 F)$ & -0.191 & 2.458 & 4.69 & $4 \%$ \\
5801.55 & 5801.51 & C IV & $(1)$ & -0.201 & 0.060 & 0.11 & $11 \%$ \\
5811.96 & 5811.98 & C IV & $(1)$ & -0.203 & 0.035 & 0.07 & $8 \%$ \\
5815.54 & 5815.97 & $\dagger$ & & -0.204 & 0.022 & 0.04 & $12 \%$ \\
5820.23 & 5820.43 & He II & $(5-34)$ & -0.205 & 0.010 & 0.02 & $24 \%$ \\
5861.56 & 5863.0 & [Mn V] & & -0.213 & 0.040 & 0.08 & $17 \%$ \\
5867.76 & 5867.82 & He II+? & Pf29 & -0.214 & 0.160 & 0.32 & $1 \%$ \\
5875.57 & 5875.67 & He I & $(11)$ & -0.216 & 15.16 & 30.41 & $6 \%$ \\
5885.42 & 5885.90 & $\dagger$ & & -0.218 & 0.062 & 0.12 & $24 \%$ \\
5912.98 & 5913.24 & He II & Pf26 & -0.223 & 0.020 & 0.04 & $40 \%$ \\
& 5931.79 & N II & $(28)$ & & & & \\
5931.69 & 5931.83 & He II & Pf25 & -0.226 & 0.025 & 0.05 & $10 \%$ \\
5952.64 & 5952.93 & He II & Pf24 & -0.229 & 0.021 & 0.04 & $20 \%$ \\
6004.40 & 6004.72 & He II & Pf22 & -0.238 & 0.028 & 0.06 & $7 \%$ \\
6024.42 & 6024.15 & P II? & $(5)$ & -0.241 & 0.010 & 0.02 & \\
6036.58 & 6036.78 & He II & Pf21 & -0.243 & 0.020 & 0.04 & $7 \%$ \\
& 6046.46 & O I & $(22)$ & & & & \\
6046.14 & 6046.26 & O I & $(22)$ & -0.245 & 0.034 & 0.07 & $12 \%$ \\
6073.74 & 6074.19 & He II & Pf20(8) & -0.249 & 0.030 & 0.07 & $16 \%$ \\
6101.50 & 6101.80 & [K IV] & $(1 F)$ & -0.254 & 0.329 & 0.74 & $13 \%$ \\
6118.47 & 6118.26 & He II & Pf19 & -0.257 & 0.019 & 0.04 & $4 \%$ \\
6138.94 & 6138.98 & S II? & $(63)$ & -0.260 & 0.016 & 0.04 & $6 \%$ \\
6156.94 & 6156.6 & C III? & $(13)$ & -0.263 & 0.023 & 0.05 & $13 \%$ \\
6161.35 & 6161.60 & [Cl II] & & -0.263 & 0.016 & 0.04 & $13 \%$ \\
6165.41 & 6166.20 & [Mn v]\# & & -0.264 & 0.019 & 0.05 & $1 \%$ \\
6170.49 & 6170.69 & He II & Pf18 & -0.265 & 0.036 & 0.09 & $24 \%$ \\
6218.42 & 6218.6 & [Mn V] & & -0.272 & 0.013 & 0.03 & $16 \%$ \\
6231.35 & line? & & & -0.274 & 0.013 & 0.03 & $31 \%$ \\
6233.60 & 6233.82 & He II & Pf17(7) & -0.275 & 0.035 & 0.09 & $11 \%$ \\
6300.00 & 6300.30 & [O I] & $(1 F)$ & -0.285 & 6.940 & 17.38 & $5 \%$ \\
6312.02 & 6312.10 & [S III] & $(3 F)$ & -0.287 & 2.666 & 6.71 & $7 \%$ \\
& & & & & & &
\end{tabular}


Table 4-Continued

\begin{tabular}{|c|c|c|c|c|c|c|c|}
\hline$\lambda(\mathrm{obs})$ & $\lambda$ (rest) & Ion & Mult. & $k_{\lambda}$ & Int(HES) & Flux(HES) & RMS \\
\hline 6346.62 & 6347.09 & Si II & $(2)$ & -0.292 & 0.055 & 0.14 & $5 \%$ \\
\hline 6363.27 & 6363.78 & {$\left[\begin{array}{ll}\mathrm{O} & \mathrm{I}\end{array}\right]$} & $(1 F)$ & -0.294 & 2.291 & 5.92 & $6 \%$ \\
\hline 6370.93 & 6371.36 & Si II & (2) & -0.295 & 0.097 & 0.25 & $8 \%$ \\
\hline 6393.80 & 6393.62 & {$[\mathrm{Mn} \mathrm{v}]$} & & -0.299 & 0.042 & 0.11 & $2 \%$ \\
\hline 6406.09 & 6406.38 & He II & $\operatorname{Pfl5}(7)$ & -0.300 & 0.056 & 0.15 & $5 \%$ \\
\hline 6462.05 & 6461.95 & $\mathrm{C}$ II & & -0.309 & 0.059 & 0.16 & $12 \%$ \\
\hline 6515.55 & line? & & & -0.316 & 0.019 & 0.05 & $2 \%$ \\
\hline 6527.62 & 6527.23 & {$[\mathrm{~N} \mathrm{II}]$} & & -0.318 & 0.066 & 0.18 & $5 \%$ \\
\hline 6544.48 & 6544.50 & $\dagger$ & & -0.320 & 0.079 & 0.22 & $5 \%$ \\
\hline 6547.90 & 6548.03 & {$[\mathrm{~N}$ II] } & $(1 F)$ & -0.321 & 14.18 & 41.98 & $2 \%$ \\
\hline 6559.81 & 6560.10 & He II & $(4-6)$ & -0.322 & 1.478 & 4.18 & $4 \%$ \\
\hline 6562.46 & 6562.82 & $\mathrm{H} \mathrm{I}$ & $\mathrm{H} \alpha$ & -0.323 & 283.92 & 803.5 & $9 \%$ \\
\hline 6577.48 & 6578.03 & $\mathrm{C}_{\mathrm{II}}$ & (2) & -0.325 & 0.263 & 0.75 & $7 \%$ \\
\hline 6580.46 & line? & & & -0.325 & 0.053 & 0.15 & $6 \%$ \\
\hline 6582.94 & 6583.45 & {$[\mathrm{~N}$ II] } & $(1 F)$ & -0.326 & 47.91 & 144.2 & $6 \%$ \\
\hline 6601.18 & 6601.10 & [Fe vII] & $(1 F)$ & -0.328 & 0.035 & 0.10 & $17 \%$ \\
\hline 6678.88 & 6678.15 & He I & $(46)$ & -0.338 & 3.275 & 9.75 & $4 \%$ \\
\hline 6683.61 & 6683.15 & He II & $\operatorname{Pf13(7)}$ & -0.339 & 0.059 & 0.18 & $15 \%$ \\
\hline 6715.89 & 6716.47 & [S II] & $(2 F)$ & -0.343 & 0.897 & 2.71 & $1 \%$ \\
\hline 6730.26 & 6730.85 & [S II] & $(2 \mathrm{~F})$ & -0.345 & 2.058 & 6.25 & $5 \%$ \\
\hline 6743.84 & line? & & & -0.346 & 0.031 & 0.09 & $3 \%$ \\
\hline 6780.04 & 6780.27 & $\mathrm{C}$ II & (14) & -0.351 & 0.027 & 0.08 & $16 \%$ \\
\hline 6787.06 & 6787.09 & $\mathrm{C}$ II & (14) & -0.351 & 0.017 & 0.05 & $22 \%$ \\
\hline 6791.09 & 6791.30 & $\mathrm{C}_{\text {II }}$ & (14) & -0.352 & 0.017 & 0.05 & $35 \%$ \\
\hline 6794.52 & 6795.00 & {$[\mathrm{~K} \mathrm{IV}]$} & $(1 F)$ & -0.352 & 0.069 & 0.21 & $20 \%$ \\
\hline 6890.37 & 6890.88 & He II & $\operatorname{PfI2(7)}$ & -0.363 & 0.077 & 0.25 & $5 \%$ \\
\hline 6894.85 & 6895.29 & $\mathrm{O} \mathrm{II} ?$ & $(45)$ & -0.364 & 0.035 & 0.11 & $4 \%$ \\
\hline 6978.44 & & line? & & -0.373 & 0.035 & 0.12 & \\
\hline 7001.66 & 7002.13 & $\mathrm{O}_{\mathrm{I}}$ & $(21)$ & -0.376 & 0.036 & 0.12 & $16 \%$ \\
\hline 7005.44 & 7005.70 & {$[\mathrm{ArV}]$} & $(1 F)$ & -0.376 & 0.297 & 1.00 & $1 \%$ \\
\hline 7064.70 & 7065.28 & He I & $(10)$ & -0.383 & 10.30 & 35.40 & \\
\hline 7135.28 & 7135.78 & {$[\mathrm{Ar} \quad \mathrm{III}]$} & $(1 F)$ & -0.390 & 16.80 & 59.16 & $4 \%$ \\
\hline 7154.34 & 7155.14 & $[\mathrm{Fe} \quad \mathrm{II}]]^{\#}$ & $(14 \mathrm{~F})$ & -0.393 & 0.033 & 0.12 & $13 \%$ \\
\hline
\end{tabular}


Table 4-Continued

\begin{tabular}{|c|c|c|c|c|c|c|c|}
\hline$\lambda(\mathrm{obs})$ & $\lambda($ rest $)$ & Ion & Mult. & $k_{\lambda}$ & $\operatorname{Int}(\mathrm{HES})$ & Flux(HES) & RMS \\
\hline 7159.86 & 7160.5 & $\mathrm{He} \mathrm{I}^{\#}$ & & -0.393 & 0.029 & 0.10 & $7 \%$ \\
\hline 7170.07 & 7170.62 & {$[\mathrm{Ar}$ IV $]$} & $(2 F)$ & -0.394 & 0.290 & 1.03 & $3 \%$ \\
\hline 7178.57 & 7177.52 & He $\mathrm{II}^{\#}$ & $?$ & -0.395 & 0.104 & 0.37 & $11 \%$ \\
\hline 7230.61 & 7231.12 & C II & (3) & -0.401 & 0.093 & 0.34 & $2 \%$ \\
\hline 7236.44 & 7236.19 & $\mathrm{C}$ II & (3) & -0.401 & 0.403 & 1.47 & $4 \%$ \\
\hline 7253.64 & 7254.38 & $\mathrm{OI}$ & (20) & -0.403 & 0.036 & 0.13 & $5 \%$ \\
\hline 7262.38 & 7262.96 & [Ar IV] & $(2 \mathrm{~F})$ & -0.404 & 0.239 & 0.88 & \\
\hline 7281.94 & 7281.35 & $\mathrm{He} \mathrm{I}$ & $(45)$ & -0.406 & 0.803 & 2.97 & $8 \%$ \\
\hline 7297.84 & 7298.05 & He I & $(1 / 9)$ & -0.407 & 0.031 & 0.11 & $22 \%$ \\
\hline 7319.37 & 7319.40 & {$\left[\begin{array}{ll}\mathrm{O} & \mathrm{II}\end{array}\right]$} & $(2 \mathrm{~F})$ & -0.410 & 8.800 & 32.96 & $5 \%$ \\
\hline 7329.54 & 7329.90 & {$\left[\begin{array}{ll}\mathrm{O} & \mathrm{II}\end{array}\right]$} & $(2 \mathrm{~F})$ & -0.411 & 7.692 & 28.91 & $4 \%$ \\
\hline 7378.17 & 7377.83 & {$[\mathrm{Ni} I \mathrm{II}]$} & $(2 F)$ & -0.416 & 0.011 & 0.04 & \\
\hline 7451.81 & 7452.5 & {$[\mathrm{Fe} \quad \mathrm{II}]^{\#}$} & $(14 F)$ & -0.423 & 0.013 & 0.05 & $7 \%$ \\
\hline 7468.05 & 7468.29 & $\mathrm{~N} \mathrm{I}$ & $(3)$ & -0.424 & 0.014 & 0.06 & $5 \%$ \\
\hline 7499.43 & 7499.84 & $\mathrm{He} \mathrm{I}$ & $(1 / 8)$ & -0.427 & 0.047 & 0.19 & $18 \%$ \\
\hline 7529.64 & 7530.83 & {$[\mathrm{Cl} \mathrm{IV}]$} & $(1 F)$ & -0.430 & 0.422 & 1.69 & $9 \%$ \\
\hline 7534.11 & 7534.83 & $\mathrm{Fe} \mathrm{II}^{\#}$ & $?$ & -0.431 & 0.027 & 0.11 & $1 \%$ \\
\hline 7592.49 & 7592.74 & He II & Pf10(6) & -0.436 & 0.200 & 0.81 & $21 \%$ \\
\hline 7750.41 & 7751.43 & {$[\mathrm{Ar} \quad \mathrm{III}]^{\#}$} & $(1 F)$ & -0.451 & 4.282 & 18.32 & $6 \%$ \\
\hline 7815.46 & 7816.16 & $\mathrm{He} I$ & $(69)$ & -0.457 & 0.080 & 0.35 & $10 \%$ \\
\hline 7821.69 & 7821.47 & S II? & (31) & -0.457 & 0.042 & 0.18 & $12 \%$ \\
\hline 7876.96 & 7875.99 & {$\left[\begin{array}{ll}\mathrm{P} & \mathrm{II}\end{array}\right]^{\#}$} & $(3 F)$ & -0.462 & 0.056 & 0.25 & $2 \%$ \\
\hline 8044.96 & 8046.27 & {$[\mathrm{Cl} \mathrm{IV}]^{\#}$} & $(1 F)$ & -0.477 & 0.939 & 4.37 & $5 \%$ \\
\hline 8186.90 & 8184.81 & $\mathrm{~N} \mathrm{I}^{\#}$ & $(2)$ & -0.488 & 0.015 & 0.07 & $21 \%$ \\
\hline 8195.70 & 8196.48 & $\mathrm{C} \mathrm{III}^{\#}$ & (43) & -0.489 & 0.316 & 1.53 & $8 \%$ \\
\hline 8203.01 & 8203.9 & He I & $(4 / 14)$ & -0.490 & 0.016 & 0.08 & $16 \%$ \\
\hline 8237.07 & 8236.78 & $\mathrm{He}$ II & Pf9 & -0.492 & 0.311 & 1.52 & $1 \%$ \\
\hline 8252.08 & 8252.50 & $\mathrm{H} \mathrm{I}$ & P39 & -0.494 & 0.041 & 0.20 & $15 \%$ \\
\hline 8254.58 & 8255.15 & $\mathrm{H} \mathrm{I}$ & P38 & -0.494 & 0.063 & 0.31 & \\
\hline 8257.53 & 8257.86 & $\mathrm{H} \mathrm{I}$ & P37 & -0.494 & 0.051 & 0.25 & $9 \%$ \\
\hline 8260.70 & 8260.94 & $\mathrm{H}$ I & P36 & -0.494 & 0.085 & 0.42 & $7 \%$ \\
\hline 8263.80 & 8264.29 & $\mathrm{H} \mathrm{I}$ & P35 & -0.495 & 0.090 & 0.44 & $2 \%$ \\
\hline 8267.20 & 8267.94 & $\mathrm{H} \mathrm{I}$ & P34 & -0.495 & 0.083 & 0.41 & $10 \%$ \\
\hline
\end{tabular}


Table 4-Continued

\begin{tabular}{|c|c|c|c|c|c|c|c|}
\hline$\lambda$ (obs) & $\lambda$ (rest) & Ion & Mult. & $k_{\lambda}$ & Int(HES) & Flux(HES) & RMS \\
\hline 8271.04 & 8271.93 & $\mathrm{H}$ I & P33 & -0.495 & 0.090 & 0.44 & \\
\hline 8275.43 & 8276.31 & $\mathrm{H} \mathrm{I}^{* *}$ & P32 & -0.495 & 0.105 & 0.52 & $4 \%$ \\
\hline 8280.24 & 8281.12 & $\mathrm{H} \mathrm{I}^{* *}$ & P31 & -0.496 & 0.120 & 0.60 & $8 \%$ \\
\hline 8285.42 & 8286.43 & $\mathrm{H} \mathrm{I}^{\#}$ & P30 & -0.496 & 0.147 & 0.73 & $10 \%$ \\
\hline 8291.17 & 8292.31 & $\mathrm{HI}^{\#}$ & $\mathrm{P} 29$ & -0.497 & 0.154 & 0.76 & $15 \%$ \\
\hline 8297.83 & 8298.84 & $\mathrm{H} \mathrm{I}^{\#}$ & P28 & -0.497 & 0.173 & 0.86 & $11 \%$ \\
\hline 8305.15 & 8306.12 & $\mathrm{HI}^{* *}$ & $\mathrm{P} 27$ & -0.498 & 0.127 & 0.63 & $6 \%$ \\
\hline 8311.31 & & line? & & -0.498 & 0.047 & 0.23 & $6 \%$ \\
\hline 8313.27 & 8314.26 & $\mathrm{H} \mathrm{I}^{\#}$ & $\mathrm{P} 26$ & -0.498 & 0.181 & 0.90 & $2 \%$ \\
\hline 8322.62 & 8323.43 & $\mathrm{H} \mathrm{I}$ & $\mathrm{P} 25$ & -0.499 & 0.194 & 0.97 & $5 \%$ \\
\hline 8333.16 & 8333.78 & $\mathrm{H} \mathrm{I}$ & $\mathrm{P} 24$ & -0.500 & 0.274 & 1.37 & $12 \%$ \\
\hline 8343.04 & $8342.2 ?$ & $\mathrm{He}^{\#}$ & $(4 / 12)$ & -0.502 & 0.054 & 0.27 & $4 \%$ \\
\hline \multirow[t]{2}{*}{8346.34} & 8345.55 & $\mathrm{H} \mathrm{I}$ & P23 & -0.502 & 0.248 & 1.25 & $6 \%$ \\
\hline & 8359.66 & He I & & & & & \\
\hline 8359.18 & 8359.01 & $\mathrm{H} \mathrm{I}$ & P22 & -0.504 & 0.247 & 1.26 & $5 \%$ \\
\hline 8361.82 & 8361.60 & $\mathrm{He} I$ & $(1 / 6)$ & -0.504 & 0.124 & 0.63 & $6 \%$ \\
\hline 8374.11 & 8374.48 & $\mathrm{H} \mathrm{I}$ & $\mathrm{P} 21$ & -0.506 & 0.261 & 1.34 & $12 \%$ \\
\hline 8391.55 & 8392.40 & $\mathrm{H} \mathrm{I}^{\#}$ & P20 & -0.509 & 0.337 & 1.74 & $3 \%$ \\
\hline 8404.98 & 8405.80 & $\mathrm{C}$ III & $6 f-8 g$ & -0.511 & 0.013 & 0.07 & $33 \%$ \\
\hline 8412.22 & 8413.32 & $\mathrm{H} \mathrm{I}$ & $\mathrm{P} 19$ & -0.512 & 0.366 & 1.91 & $1 \%$ \\
\hline 8421.30 & 8420.97 & O I & $(54)$ & -0.514 & 0.022 & 0.11 & $3 \%$ \\
\hline 8432.63 & 8433.85 & {$[\mathrm{Cl} \quad \mathrm{III}]^{\#}$} & $(3 F)$ & -0.515 & 0.052 & 0.27 & $6 \%$ \\
\hline 8436.96 & 8437.96 & $\mathrm{H} \mathrm{I}^{\#}$ & P18 & -0.516 & 0.412 & 2.17 & $7 \%$ \\
\hline 8443.57 & 8444.4 & $\mathrm{He} \mathrm{I}^{\#}$ & $(4 / 11)$ & -0.517 & 0.025 & 0.13 & \\
\hline 8445.59 & 8446.48 & $\mathrm{OI}^{\#}$ & (4) & -0.517 & 0.424 & 2.25 & $5 \%$ \\
\hline 8450.47 & 8451.55 & $\mathrm{SI}^{\#}$ & $(14)$ & -0.518 & 0.025 & 0.13 & $18 \%$ \\
\hline 8468.17 & 8467.26 & $\mathrm{H} \mathrm{I}$ & P17 & -0.521 & 0.453 & 2.43 & $20 \%$ \\
\hline 8481.23 & 8481.16 & [Cl III] & $(3 F)$ & -0.523 & 0.047 & 0.25 & $8 \%$ \\
\hline 8486.41 & 8485.8 & He $\mathrm{I}$ & & -0.524 & 0.022 & 0.12 & \\
\hline 8488.83 & 8488.77 & He I & & -0.524 & 0.022 & 0.12 & $28 \%$ \\
\hline 8499.56 & 8500.00 & {$[\mathrm{Cl} \mathrm{III}]$} & $(3 F)$ & -0.526 & 0.049 & 0.27 & $4 \%$ \\
\hline 8502.00 & 8502.49 & $\mathrm{H} \mathrm{I}_{\mathrm{I}}$ & P16 & -0.526 & 0.517 & 2.82 & \\
\hline 8527.91 & 8528.99 & He I\# & $6 / 15,10 / 17$ & -0.530 & 0.023 & 0.13 & $8 \%$ \\
\hline
\end{tabular}


Table 4-Continued

\begin{tabular}{|c|c|c|c|c|c|c|c|}
\hline$\lambda(\mathrm{obs})$ & $\lambda($ rest $)$ & Ion & Mult. & $k_{\lambda}$ & Int(HES) & Flux(HES) & RMS \\
\hline 8544.21 & 8545.38 & $\mathrm{H} \mathrm{I}$ & $\mathrm{P} 15$ & -0.532 & 0.673 & 3.74 & $15 \%$ \\
\hline 8577.93 & 8578.70 & {$\left[\begin{array}{lll}\mathrm{Cl} & \mathrm{II}\end{array}\right]^{\#}$} & $(1 \mathrm{~F})$ & -0.537 & 0.195 & 1.10 & $18 \%$ \\
\hline 8581.53 & 8581.70 & He I & $(6 / 14)$ & -0.538 & 0.087 & 0.49 & $30 \%$ \\
\hline 8599.21 & 8598.39 & $\mathrm{H} \mathrm{I}^{\#}$ & $\mathrm{P} 14$ & -0.540 & 0.781 & 4.46 & $4 \%$ \\
\hline 8617.08 & 8616.96 & {$[\mathrm{Fe} \mathrm{II}]$} & $(13 F)$ & -0.543 & 0.040 & 0.23 & $12 \%$ \\
\hline 8647.30 & 8648.26 & He I & $(6 / 13)$ & -0.547 & 0.042 & 0.24 & $10 \%$ \\
\hline 8660.86 & 8661.40 & He II & $(6-26)$ & -0.549 & 0.039 & 0.23 & $7 \%$ \\
\hline 8663.85 & 8665.02 & $\mathrm{H} \mathrm{I}^{\#}$ & $\mathrm{P} 13$ & -0.550 & 1.046 & 6.15 & $14 \%$ \\
\hline 8728.03 & 8727.13 & {$[\mathrm{Cl}]^{\#}$} & & -0.559 & 0.140 & 0.85 & $12 \%$ \\
\hline 8734.05 & 8733.43 & $\mathrm{He} \mathrm{I}$ & $(6 / 12)$ & -0.560 & 0.031 & 0.19 & $5 \%$ \\
\hline 8750.42 & 8750.48 & $\mathrm{H} \mathrm{I}$ & $\mathrm{P} 12$ & -0.562 & 0.984 & 6.03 & $5 \%$ \\
\hline 8776.14 & 8776.77 & $\mathrm{He} \mathrm{I}$ & $(4 / 9)$ & -0.566 & 0.065 & 0.40 & $10 \%$ \\
\hline 8788.92 & 8787.60 & {$\left[\mathrm{P}_{\mathrm{I}}\right]^{\#}$} & & -0.568 & 0.026 & 0.16 & \\
\hline 8797.72 & 8798.90 & $\mathrm{He} \mathrm{II}^{\#}$ & $(6-23)$ & -0.569 & 0.020 & 0.13 & $19 \%$ \\
\hline 8844.67 & 8845.38 & He I & $(6 / 11)$ & -0.575 & 0.068 & 0.44 & $18 \%$ \\
\hline 8847.67 & 8848.05 & $\mathrm{He} \mathrm{I}$ & $(7 / 11)$ & -0.576 & 0.035 & 0.22 & $7 \%$ \\
\hline 8863.84 & 8862.79 & $\mathrm{H} \mathrm{I}$ & P11 & -0.578 & 1.502 & 9.68 & $16 \%$ \\
\hline 8926.29 & 8926.06 & Mn I? & $(56)$ & -0.587 & 0.098 & 0.65 & $21 \%$ \\
\hline 8929.47 & 8929.00 & He II & $(6-21)$ & -0.587 & 0.011 & 0.07 & $37 \%$ \\
\hline 8985.83 & & line? & & -0.595 & 0.025 & 0.17 & $10 \%$ \\
\hline 8996.25 & 8996.99 & He I & $(6 / 10)$ & -0.596 & 0.079 & 0.54 & $22 \%$ \\
\hline 8998.45 & 8999.75 & $\mathrm{He} \mathrm{I}^{\#}$ & $(7 / 10)$ & -0.596 & 0.063 & 0.43 & $6 \%$ \\
\hline 9000.79 & 8999.75 & $\mathrm{He} \mathrm{I}^{\#}$ & $(7 / 10)$ & -0.597 & 0.043 & 0.29 & $27 \%$ \\
\hline 9015.42 & 9014.91 & $\mathrm{H}_{1}$ & P10 & -0.599 & 1.510 & 10.40 & $32 \%$ \\
\hline 9062.21 & 9062.53 & $\mathrm{C}_{\mathrm{I}}$ & (3) & -0.605 & 0.064 & 0.45 & $27 \%$ \\
\hline 9067.88 & 9068.90 & {$[\mathrm{~S}$ III $]$} & $(1 F)$ & -0.606 & 22.34 & 157.4 & $13 \%$ \\
\hline 9122.70 & 9123.60 & {$[\mathrm{Cll} I]^{\#}$} & $(1 F)$ & -0.610 & 0.053 & 0.38 & $39 \%$ \\
\hline 9209.25 & 9210.28 & $\mathrm{He} \mathrm{I}^{\#}$ & $(83), 6 / 9$ & -0.612 & 0.111 & 0.79 & $39 \%$ \\
\hline 9212.03 & 9212.53 & $\mathrm{~S} I ?$ & (1) & -0.612 & 0.034 & 0.24 & $45 \%$ \\
\hline 9213.80 & 9213.24 & $\mathrm{He} \mathrm{I}$ & $(7 / 9)$ & -0.612 & 0.037 & 0.27 & $38 \%$ \\
\hline 9222.03 & & line? & & -0.612 & 0.054 & 0.39 & $40 \%$ \\
\hline 9223.84 & & line? & & -0.612 & 0.038 & 0.28 & $47 \%$ \\
\hline 9227.66 & 9227.70 & $\mathrm{He} \mathrm{I}$ & $(5 / 9)$ & -0.612 & 3.287 & 23.66 & $34 \%$ \\
\hline
\end{tabular}


Table 4-Continued

\begin{tabular}{|c|c|c|c|c|c|c|c|}
\hline$\lambda(\mathrm{obs})$ & $\lambda$ (rest) & Ion & Mult. & $k_{\lambda}$ & $\operatorname{Int}(\mathrm{HES})$ & Flux (HES) & $\mathrm{RMS}$ \\
\hline 9231.21 & 9229.02 & $\mathrm{H} \mathrm{I}^{\#}$ & P9 & -0.612 & 0.022 & 0.16 & $42 \%$ \\
\hline 9373.49 & 9373.28 & $\mathrm{Ne} I$ & (33) & -0.616 & 0.162 & 1.18 & $50 \%$ \\
\hline 9464.32 & 9463.57 & $\mathrm{He} \mathrm{I}$ & $(1 / 5)$ & -0.618 & 0.264 & 1.94 & $28 \%$ \\
\hline 9515.39 & 9516.5 & $\mathrm{He} \mathrm{I}^{\#}$ & $(4 / 7)$ & -0.619 & 0.114 & 0.84 & $47 \%$ \\
\hline 9524.87 & 9526.0 & $\mathrm{He} \mathrm{I} \#$ & $(6 / 8)$ & -0.620 & 0.135 & 1.00 & $39 \%$ \\
\hline 9529.78 & $9531.0 ?$ & {$[S \text { III] }]^{\#}$} & $(1 F)$ & -0.620 & 65.68 & 484.2 & $21 \%$ \\
\hline 9544.65 & 9545.97 & $\mathrm{H} \mathrm{I}$ & P8 & -0.620 & 3.140 & 23.17 & $42 \%$ \\
\hline 9691.00 & & line? & & -0.624 & 0.086 & 0.64 & $15 \%$ \\
\hline 9823.23 & 9824.11 & {$[\mathrm{C} \text { I }]^{\#}$} & $(1 F)$ & -0.627 & 0.100 & 0.76 & $12 \%$ \\
\hline 9848.76 & 9850.24 & {$\left[\begin{array}{ll}\mathrm{C} & \mathrm{I}\end{array}\right]^{\#}$} & $(1 \mathrm{~F})$ & -0.627 & 0.272 & 2.06 & $8 \%$ \\
\hline 9902.19 & 9902.70 & [K III] & $(1 F)$ & -0.628 & 0.148 & 1.12 & $9 \%$ \\
\hline 10026.2 & 10027.7 & $\mathrm{He} \mathrm{I} \#$ & $(6 / 7)$ & -0.631 & 0.194 & 1.48 & $11 \%$ \\
\hline 10029.6 & 10027.6 & $\mathrm{He} \mathrm{I}^{\#}$ & $(6 / 7)$ & -0.631 & 0.071 & 0.54 & $23 \%$ \\
\hline 10043.5 & 9545.9 & He II $\#$ & $(6-14)$ & -0.631 & 0.052 & 0.40 & $20 \%$ \\
\hline 10047.7 & 10049.4 & $\mathrm{H} \mathrm{I}^{\#}$ & P7 & -0.631 & 4.808 & 36.81 & $3 \%$ \\
\hline 10125.6 & 10123.6 & $\mathrm{He} \mathrm{II}^{\#}$ & $(4-5)$ & -0.633 & 0.191 & 1.47 & $32 \%$ \\
\hline 10321.3 & 10320.6 & {$[\mathrm{~S} \quad \mathrm{II}]^{\#}$} & & -0.637 & 0.378 & 2.95 & $45 \%$ \\
\hline 10336.3 & 10338.8 & {$[\mathrm{~S} \text { II }]^{\#}$} & & -0.638 & 0.249 & 1.95 & $47 \%$ \\
\hline
\end{tabular}

$\dagger$ These unidentified lines are seen in other PNe, e.g. IC 4997 and NGC 7662.

? Unlikely identification.

\# Identification with too large a wavelength discrepancy.

** Lines affected by telluric absorption lines. Some lines may be lost in the telluric absroption region, e.g. $7595-7700 \AA$. The spectrum of IC 5117 was not divided by that of a standard star to take out the first order effects of the atmosphere.

References. - See Hyung \& Aller (1996) for the identifications and references therein. 
Table 5. Diagnostic Line Ratios suitable for fixing $N_{\epsilon}, \mathrm{T}_{\epsilon}$.

\begin{tabular}{|c|c|c|c|c|}
\hline Ion & Lines & Ratio & Determines & Remarks \\
\hline$[\mathrm{N}$ I $]$ & $\mathrm{I}(\lambda 5198) / \mathrm{I}(\lambda 5200)$ & 1.72 & $N_{\epsilon}$ & \\
\hline$[\mathrm{N}$ II $]$ & $\mathrm{I}(\lambda 6548+\lambda 6583) / \mathrm{I}\left(\lambda 5755^{a}\right)$ & 25.3 & $\mathrm{~T}_{\epsilon}$ & \\
\hline$\left[\begin{array}{lll}\mathrm{O} & \mathrm{II}\end{array}\right]$ & $\mathrm{I}(\lambda 3726) / \mathrm{I}(\lambda 3729)$ & 3.10 & $N_{\epsilon}$ & N/A? \\
\hline$[\mathrm{O} \mathrm{II}]$ & $\mathrm{I}(\lambda 3726+\lambda 3729) / \mathrm{I}(\lambda 7319 / 20+\lambda 7329 / 30)$ & 0.695 & $N_{\epsilon}, \mathrm{T}_{\epsilon}$ & {$[\mathrm{O}$ II $] \mathrm{a}$} \\
\hline$\left[\begin{array}{ll}O & \text { III }\end{array}\right]$ & $\mathrm{I}(\lambda 4959+\lambda 5007) / \mathrm{I}(\lambda 4363)$ & 88.3 & $\mathrm{~T}_{\epsilon}$ & \\
\hline$[\mathrm{Cl} \mathrm{III}]^{a}$ & $\mathrm{I}(\lambda 5518) / \mathrm{I}(\lambda 5538)$ & 0.328 & $N_{\epsilon}$ & \\
\hline [Cl IV] & $\mathrm{I}(\lambda 7530+\lambda 8045) / \mathrm{I}(\lambda 5323)$ & 30.9 & $\mathrm{~T}_{\epsilon}$ & \\
\hline [Ar III] & $\mathrm{I}(\lambda 7136+\lambda 7751) / \mathrm{I}\left(\lambda 5191^{a}\right)$ & 135.1 & $\mathrm{~T}_{\epsilon}$ & \\
\hline [Ar Iv] & $\mathrm{I}(\lambda 4740+\lambda 4711) / \mathrm{I}(\lambda 7171)$ & 20.2 & $N_{\epsilon}, \mathrm{T}_{\epsilon}$ & $\mathrm{N} / \mathrm{A} ?$ \\
\hline$[\mathrm{S} I \mathrm{II}]$ & $\mathrm{I}\left(\lambda 6716^{a}+\lambda 6731\right) / \mathrm{I}\left(\lambda 4069^{a}\right)$ & 0.975 & $N_{\epsilon}, \mathrm{T}_{\epsilon}$ & {$[\mathrm{S}$ II $] \mathrm{a}$} \\
\hline$[\mathrm{S}$ II $]$ & $I\left(\lambda 6716^{a}\right) / I(\lambda 6731)$ & 0.436 & $N_{\epsilon}$ & $\mathrm{N} / \mathrm{A} ?$ \\
\hline$[\mathrm{S} I \mathrm{III}]$ & $\mathrm{I}\left(\lambda 9069+\lambda 9531^{a}\right) / \mathrm{I}(\lambda 6312)$ & 33.0 & $\mathrm{~T}_{\epsilon}$ & \\
\hline
\end{tabular}

${ }^{a}$ relatively weak line, or of poor quality.

Note. - N/A ?: diagnostic informations is useless, or not in a reasonable range (due to observational errors, or poor measurements). 
Table 6. Model Details for IC 5117.

\begin{tabular}{ll}
\hline Parameter & Model B \\
\hline $\mathrm{R}_{\text {in }}(\mathrm{pc})$ & 0.008 \\
$\mathrm{R}_{\text {out }}(\mathrm{pc})^{a}$ & $0.0154\left(\triangle \theta \sim 1.06^{\prime \prime}\right)$ \\
$N_{H}\left(\mathrm{~cm}^{-3}\right)$ & 80000 \\
& \\
DISTANCE $=$ & $3000 \mathrm{pc}$ \\
$\mathrm{M}_{\text {dust }} / \mathrm{M}_{\text {gas }}=$ & 0.001 \\
$\mathrm{~F}(\mathrm{H} \beta)-\mathrm{obs}=$ & $8.33 \sim 10.7(-11) \mathrm{erg} \mathrm{cm}^{-2} \mathrm{~s}^{-1}$ \\
$\mathrm{~F}(\mathrm{H} \beta)-\mathrm{pred}=$ & $9.67(-11) \mathrm{erg} \mathrm{cm} \mathrm{cm}^{-2} \mathrm{~s}^{-1}$ \\
$\mathrm{CSPN} \mathrm{T}(\star)^{c}=$ & $120000 \mathrm{~K}(\log \mathrm{g}=8.5)$ \\
$\mathrm{CSPN} \mathrm{R}(\star)=$ & $0.16 \mathrm{R} \odot\left(\mathrm{L}(\star)=5000 \mathrm{~L}_{\odot}\right)$ \\
$\mathrm{T}_{\epsilon}([\mathrm{O} \mathrm{II}, \mathrm{III}, \mathrm{IV}])$ & $12500,11800,13500 \mathrm{~K}$ \\
$\mathrm{Magnitude}$ & $\mathrm{V}_{\text {pred }}=15.1 \& \mathrm{~V}_{\text {obs }}=14.0^{d}$ \\
\hline
\end{tabular}

a density bounded.

${ }^{b}$ extinction corrected with $C=1.29 \sim 1.4$.

c Hubeny non-LTE model atmosphere. See text.

${ }^{\mathrm{d}}$ corrected with $\mathrm{E}(\mathrm{B}-\mathrm{V})=0.88$. 
Table 7. Comparison of observed and predicted intensities for IC 5117.

\begin{tabular}{|c|c|c|c|c|c|}
\hline El-ion & $\lambda$ & $\mathrm{I}(\mathrm{AC} 79)^{a}$ & $\mathrm{I}_{\mathrm{obs}}{ }^{b}$ & I(Model-A) & I(Model-B) \\
\hline \multirow[t]{3}{*}{$\mathrm{He} \mathrm{I}$} & 5876 & 14.79 & 15.16 & 14.61 & 15.49 \\
\hline & 6678 & 2.82 & 3.28 & 3.72 & 3.81 \\
\hline & 4471 & 4.36 & 5.30 & 5.09 & 5.41 \\
\hline \multirow[t]{3}{*}{ He II } & 4686 & 9.33 & 9.44 & 9.99 & 7.63 \\
\hline & 5412 & 0.74 & 0.92 & 0.81 & 0.64 \\
\hline & 1640 & & {$[58.8]$} & 68.6 & 55.3 \\
\hline \multirow[t]{2}{*}{ C II } & $2325 / 28$ & & {$[83.0]$} & 53.0 & 60.8 \\
\hline & 4267 & 0.43 & 0.48 & 0.47 & 0.27 \\
\hline C III & $1907 / 09$ & & [418.0] & 401.2 & 415.3 \\
\hline C IV & $1548 / 51$ & & [126.9] & 209.0 & 409.9 \\
\hline \multirow[t]{3}{*}{$\mathrm{N}$ II } & 6584 & 38.09 & 50.61 & 45.85 & 41.96 \\
\hline & 6548 & 12.88 & 14.18 & 15.83 & 14.49 \\
\hline & 5755 & 2.04 & 2.46 & 1.97 & 3.17 \\
\hline N III & $1747-52$ & & {$[12.4]$} & 17.8 & 17.5 \\
\hline N IV & $1483 / 86$ & & $\cdots$ & 6.51 & 12.23 \\
\hline \multirow[t]{4}{*}{ O II } & 3726 & $11.1^{c}$ & 8.67 & 7.70 & 7.24 \\
\hline & 3729 & $3.59^{c}$ & 2.80 & 2.77 & 2.55 \\
\hline & $7321 / 2$ & $6.57^{c}$ & 8.80 & 3.63 & 7.22 \\
\hline & $7332 / 3$ & $5.73^{c}$ & 7.69 & 2.92 & 5.80 \\
\hline \multirow[t]{4}{*}{ O III } & $1660 / 66$ & & {$[38.0]$} & 16.6 & 27.4 \\
\hline & 4363 & 19.95 & 19.72 & 13.31 & 20.17 \\
\hline & 4959 & 524.8 & 395.0 & 452.8 & 481.5 \\
\hline & 5007 & 1479 & 1346 & 1304 & 1387 \\
\hline \multirow[t]{2}{*}{$\mathrm{Ne}$ III } & 3868 & 154.9 & 103.8 & 132.0 & 135.5 \\
\hline & 3969 & 61.66 & 42.64 & 39.38 & 40.43 \\
\hline \multirow[t]{2}{*}{ Ne IV } & $2422 / 25$ & & [11.1] & 3.39 & 5.56 \\
\hline & $4725 / 27$ & 0.27 & 0.11 & 0.03 & 0.10 \\
\hline \multirow[t]{4}{*}{ S II } & 4068 & 3.47 & 3.03 & 1.64 & 3.06 \\
\hline & 4076 & 1.07 & 1.13 & 0.55 & 1.01 \\
\hline & 6717 & 0.58 & 0.90 & 0.32 & 0.30 \\
\hline & 6731 & 1.29 & 2.06 & 0.70 & 0.66 \\
\hline \multirow[t]{2}{*}{ S III } & 6312 & 2.04 & 2.67 & 2.29 & 2.31 \\
\hline & 9069 & $\ldots$ & 22.34 & 33.52 & 25.26 \\
\hline
\end{tabular}


Table 7-Continued

\begin{tabular}{llcccc}
\hline \hline El-ion & $\lambda$ & $\mathrm{I}^{2}(\mathrm{AC79})^{a}$ & $\mathrm{I}_{\text {obs }}{ }^{b}$ & $\mathrm{I}($ Model-A) & I(Model-B) \\
\hline \multirow{3}{*}{ S IV } & 9531 & $\ldots$ & 65.68 & 81.66 & 61.53 \\
Cl III & $10.5 \mu \mathrm{m}$ & $33.6^{d}$ & $\ldots$ & 73.21 & 31.4 \\
& 5518 & 0.083 & 0.14 & 0.15 & 0.08 \\
Cl IV & 5538 & 0.29 & 0.43 & 0.50 & 0.31 \\
& 7530 & 0.27 & 0.42 & 0.42 & 0.47 \\
Ar III & 8046 & 0.66 & 0.94 & 0.97 & 1.10 \\
& 5193 & 0.15 & 0.16 & 0.14 & 0.14 \\
& 7136 & 14.13 & 16.80 & 16.36 & 12.41 \\
Ar IV & 7751 & 3.24 & 4.28 & 3.95 & 3.00 \\
& 4711 & 1.84 & 1.13 & 2.42 & 1.86 \\
& 4740 & 5.13 & 4.72 & 8.52 & 8.96 \\
& 7238 & 0.25 & $\ldots$ & 0.16 & 0.24 \\
& 7263 & 0.12 & 0.24 & 0.17 & 0.10 \\
Ar V & 7171 & 0.16 & 0.29 & 0.12 & 0.20 \\
& 6435 & 0.03 & $\ldots$ & 0.12 & 0.30 \\
K IV & 7005 & 0.16 & 0.30 & 0.26 & 0.64 \\
Si III & 6102 & 0.19 & 0.33 & 0.33 & 0.33 \\
& $1883 / 92$ & & {$[41.7]$} & 44.66 & 45.53 \\
\hline
\end{tabular}

a Aller \& Czyzak (1979, AC79).

${ }^{b}\left[\mathrm{I}_{\mathrm{obs}}\right]$ : Intensities in square brackets are from the low resolution IUE data.

c Calculated using the HES line ratios. ITS spectral resolution $\sim 2 \AA$.

d [S IV] measurement from Beck et al. (1981), and intensity is obtained assuming $\mathrm{I}(\mathrm{H} \beta)=8.33(-11) \mathrm{erg} \mathrm{cm}^{-2} \mathrm{~s}^{-1}$ [See Table 6]. 
Table 8. Fractional ionic concentration.

\begin{tabular}{|c|c|c|c|c|}
\hline Ion & Wavelength & Intensity & $\frac{N(\text { ion })}{N\left(H^{+}\right)}$ & $\sum \frac{N(i o n)}{N\left(H^{+}\right)}$ \\
\hline \multirow[t]{2}{*}{ He I } & 6678 & 3.275 & $7.84(-2)$ & \\
\hline & 5876 & 15.16 & $8.87(-2)$ & \\
\hline \multirow[t]{2}{*}{ He II } & 4686 & 9.44 & $8.13(-3)$ & \\
\hline & 5412 & 0.92 & $5.84(-3)$ & $9.84(-2)$ \\
\hline $\mathrm{C}$ II & $2325 / 28$ & 83 & $4.82(-5)$ & \\
\hline $\mathrm{C}$ III & $1907 / 09$ & 418 & $3.44(-4)$ & \\
\hline $\mathrm{C} \mathrm{IV}$ & $1549 / 51$ & 127 & $8.19(-5)$ & $4.74(-4)$ \\
\hline $\mathrm{N}$ II & $6548 / 84,5755$ & $14.18,47.91,2.46$ & $1.08(-5), 1.26(-5), 9.07(-6)$ & \\
\hline $\mathrm{N}$ III & $1753-$ & 12 & $4.54(-5)$ & $5.75(-5)$ \\
\hline $\mathrm{O}_{\mathrm{I}}$ & $6300 / 63$ & $6.94,2.29$ & $6.79(-6), 7.04(-6)$ & \\
\hline $\mathrm{O}$ II & $3727 / 29,7320 / 30$ & $8.67,2.80,8.80,7.69$ & $\begin{array}{r}3.03(-5), 2.78(-5), 4.13(-5), 6.89(-5) \\
1.05(-5), 0.94(-5), 2.76(-5), 4.62(-5)^{a}\end{array}$ & \\
\hline $\mathrm{O}$ III & $4957,5007,4363$ & $395,1346,19.7$ & $2.84(-4), 3.33(-4), 3.36(-4)$ & $3.73(-4)$ \\
\hline $\mathrm{O}$ III & $1660-$ & 38 & $4.92(-4)^{b}$ & \\
\hline Ne III & $3869 / 3967$ & $103.8,42.6$ & $4.54(-5), 6.26(-5)$ & \\
\hline Ne IV & 2422 & 11 & $1.27(-5)$ & $6.31(-5)$ \\
\hline $\mathrm{S}$ II & $6717 / 31,4068$ & $0.90,2.06,3.03$ & $7.23(-7), 7.48(-7), 2.31(-7)$ & \\
\hline $\mathrm{S}$ III & $6312,9069,9531$ & $2.67,22.34,65.68$ & $3.12(-6), 2.26(-6), 2.73(-6)$ & \\
\hline S IV & $10.5 \mu \mathrm{m}$ & 33.6 & $4.26(-6)$ & $7.37(-6)$ \\
\hline Ar III & $7135,7751,5192$ & $16.8,4.28,0.16$ & $9.81(-7), 1.04(-6), 8.67(-7)$ & \\
\hline Ar IV & $4711 / 40,7263,7171$ & $1.13,4.72,0.24,0.29$ & $5.52(-7), 5.01(-7), 1.14(-6)^{b}, 1.10(-6)^{b}$ & \\
\hline $\operatorname{Ar} v$ & 7006 & 0.30 & $3.54(-8)$ & $1.59(-6)$ \\
\hline $\mathrm{Cl}$ III & $5517 / 37$ & $0.14,0.43$ & $7.59(-8), 5.91(-8)$ & \\
\hline $\mathrm{Cl} \mathrm{IV}$ & $7530 / 8045,5323$ & $0.42,0.94,0.044$ & $6.38(-8), 6.12(-8), 5.90(-8)$ & $1.25(-7)$ \\
\hline $\mathrm{K} \mathrm{IV}$ & 6102 & 0.329 & $5.94(-8)$ & $5.94(-8)$ \\
\hline
\end{tabular}


Table 8-Continued

\begin{tabular}{lrrrr}
\hline \hline Ion & Wavelength & Intensity & $\frac{N(\text { ion })}{N\left(H^{+}\right)}$ & $\Sigma \frac{N(\text { ion })}{N\left(H^{+}\right)}$ \\
\hline Si III & $1882 / 92$ & 41.7 & $7.52(-6)$ & $7.52(-6)$ \\
\hline
\end{tabular}

aderivation with $N_{\epsilon}=40000 \mathrm{~cm}^{-3}$ and $\mathrm{T}_{\epsilon}=13000$ $\mathrm{K}$ (see text).

${ }^{b}$ ignored because of relatively weak line intensities.

Note. $-\mathrm{X}(-\mathrm{Y})$ implies $\mathrm{X} \times 10^{-Y}$. Ionic concentrations are derived with $N_{\epsilon}=90000 \mathrm{~cm}^{-3}$ and $\mathrm{T}_{\epsilon}=12000$ $\mathrm{K}$. 
Table 9. Comparison of ICF and Model abundances for IC 5117.

\begin{tabular}{lccccccccc}
\hline \hline \multicolumn{1}{c}{ Elem. } & $\Sigma \frac{N(\text { ion })}{N\left(H^{+}\right)}$ & ICF & $N($ ICF $)$ & $N($ Model) & $\triangle$ & IC 5117 & AC83 $^{a}$ & Mean $^{b}$ & SUN $^{c}$ \\
\hline He I, II & $9.84(-2)$ & 1.02 & $1.00(-1)$ & $9.86(-2)$ & 0.01 & 0.10 & 0.11 & 0.11 & 0.1 \\
C II, III, IV & $4.74(-4)$ & 1.02 & $4.83(-4)$ & $6.00(-4)$ & -0.09 & $5.00(-4)$ & $8.9(-4)$ & $6.48(-4)$ & $3.55(-4)$ \\
N II, III & $5.75(-5)$ & 1.92 & $1.10(-4)$ & $1.40(-4)$ & -0.10 & $1.30(-4)$ & $1.31(-4)$ & $1.40(-4)$ & $9.33(-5)$ \\
O I, II, III & $3.73(-4)$ & 1.04 & $3.88(-4)$ & $3.75(-4)$ & 0.01 & $3.80(-4)$ & $3.75(-4)$ & $4.93(-4)$ & $7.41(-4)$ \\
Ne III, IV & $6.31(-5)$ & 1.02 & $6.44(-5)$ & $6.50(-5)$ & -0.00 & $6.50(-5)$ & $9.31(-5)$ & $1.25(-4)$ & $1.17(-4)$ \\
S II, III, IV & $7.37(-6)$ & 1.21 & $8.85(-6)$ & $9.00(-6)$ & -0.01 & $8.90(-6)$ & $1.21(-5)$ & $8.08(-6)$ & $1.62(-5)$ \\
Ar III, IV, V & $1.59(-6)$ & 1.01 & $1.61(-6)$ & $2.00(-6)$ & -0.09 & $1.80(-6)$ & $2.07(-6)$ & $2.42(-6)$ & $3.98(-6)$ \\
Cl III, IV & $1.25(-7)$ & 1.55 & $1.94(-7)$ & $1.70(-7)$ & 0.06 & $1.85(-7)$ & $1.43(-7)$ & $1.66(-7)$ & $3.88(-7)$ \\
K IV & $5.94(-8)$ & 1.11 & $6.59(-8)$ & $6.50(-8)$ & 0.00 & $6.50(-8)$ & $3.3(-8)$ & $\ldots$ & $1.35(-7)$ \\
Si III & $7.52(-6)$ & 6.67 & $5.01(-5)$ & $5.00(-5)$ & 0.00 & $5.00(-5)$ & $\ldots$ & $\ldots$ & $3.55(-5)$ \\
& & & & & & & & &
\end{tabular}

a Aller \& Czyzak (1983, AC83).

b Average (or normal) abundances by Kingsburgh \& Barlow (1994) and Aller \& Czyzak (1983)

c Solar abundances from Grevesse and Noels (1993).

Note. $-\mathrm{X} 1, \mathrm{X} 2(-\mathrm{Y})$ implies $\mathrm{X} 1 \times 10^{-Y}, \mathrm{X} 2 \times 10^{-Y}$. All abundances are given relative to $N\left(\mathrm{H}^{+}\right) . \triangle$ : the logarithmic difference, i.e., $\log N(\mathrm{ICF})-\log N$ (Model), which is less than 0.1 dex for most elements. Our derivation agrees with AC83 to within a factor of $2(<0.3 \mathrm{dex})$. 
